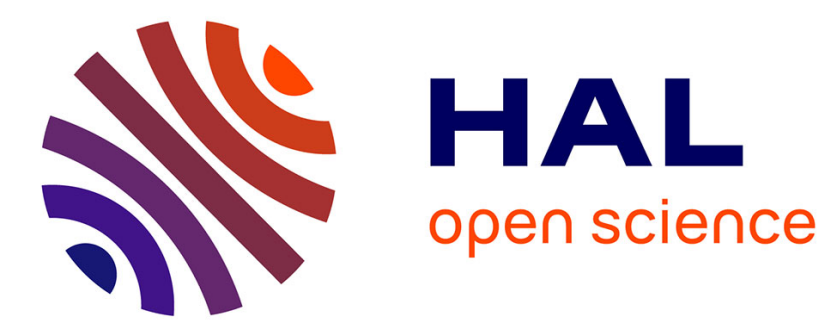

\title{
Traduire la règle, trahir la loi : la traduction de dix poèmes extraits de Voies de disparition de Simon Jardin
}

Amélie Derome, Simon Jardin

\section{To cite this version:}

Amélie Derome, Simon Jardin. Traduire la règle, trahir la loi : la traduction de dix poèmes extraits de Voies de disparition de Simon Jardin. Trans-action, 2019, 10.4000/trans.2699 . hal-03094823

\section{HAL Id: hal-03094823 \\ https://hal.science/hal-03094823}

Submitted on 4 Jan 2021

HAL is a multi-disciplinary open access archive for the deposit and dissemination of scientific research documents, whether they are published or not. The documents may come from teaching and research institutions in France or abroad, or from public or private research centers.
L'archive ouverte pluridisciplinaire HAL, est destinée au dépôt et à la diffusion de documents scientifiques de niveau recherche, publiés ou non, émanant des établissements d'enseignement et de recherche français ou étrangers, des laboratoires publics ou privés. 


\section{TRANS-}

Revue de littérature générale et comparée

2019

Hors la loi ( $\left.\mathrm{N}^{\circ} 25 \mid 2020\right)$

\section{Traduire la règle, trahir la loi : la traduction de dix poèmes extraits de Voies de disparition de Simon Jardin}

Translating the rules and betraying the law: translating ten poems from Voies de disparition by Simon Jardin

Traducir las reglas y traicionar la ley: traducir diez poemas de Voies de disparition de Simon Jardin

\section{Amélie Derome et Simon Jardin}

\section{OpenEdition}

Journals

Édition électronique

URL : http://journals.openedition.org/trans/2699

DOI : $10.4000 /$ trans.2699

ISSN : $1778-3887$

Éditeur

Presses Sorbonne Nouvelle

Ce document vous est offert par Aix-Marseille Université (AMU)

\section{Aix $*$ Marseille}

\section{universite}

Référence électronique

Amélie Derome et Simon Jardin, « Traduire la règle, trahir la loi : la traduction de dix poèmes extraits de Voies de disparition de Simon Jardin », TRANS- [En ligne], | 2019, mis en ligne le 01 décembre 2019 consulté le 04 janvier 2021. URL : http://journals.openedition.org/trans/2699 ; DOI : https://doi.org/ $10.4000 /$ trans.2699

Ce document a été généré automatiquement le 4 janvier 2021.

Tous droits réservés 


\title{
Traduire la règle, trahir la loi : la traduction de dix poèmes extraits de Voies de disparition de Simon Jardin
}

\author{
Translating the rules and betraying the law: translating ten poems from Voies \\ de disparition by Simon Jardin \\ Traducir las reglas y traicionar la ley: traducir diez poemas de Voies de \\ disparition de Simon Jardin
}

Amélie Derome et Simon Jardin

1 «Poetry is what gets lost in translation ${ }^{1}$ ", disait Robert Frost. Si la sentence du poète américain tombe comme un couperet, l'idée qui la sous-tend est tenace. Dante estimait également qu' " aucune chose de celles qui ont été mises en harmonie par lien de poésie ne peut se transporter de sa langue en une autre sans qu'on rompe sa douceur et son harmonie, et c'est la raison pourquoi Homère ne doit pas être mis du grec en latin ${ }^{2}$. » En effet, la pensée dominante de la traduction de la poésie conclut à son impossibilité. D'aucuns ont tâché d'opposer un argument pratique à ce verdict : les épopées d'Homère comptent parmi les textes les plus traduits de l'histoire de la littérature et les hommes n'ont eu de cesse de traduire la poésie. Une autre contre-attaque s'est organisée en attirant l'attention sur les grandes traductions canoniques, elles-mêmes œuvres de poètes, à l'image du Poe de Baudelaire ${ }^{3}$, ou de l'Iliade de Pope ${ }^{4}$. Les tenanciers de l'impossibilité de la traduction de la poésie cédèrent alors une concession à leurs adversaires : traduire la poésie devint possible à la condition d'être poète soi-même.

2 Le débat s'est ensuite porté sur la rime et les difficultés qu'elle engendre à la traduction. Comment la rendre, en effet, sans risquer de s'éloigner drastiquement du sens du texte ? À l'inverse, sa suppression ne constituerait-elle pas une atteinte grave à la forme du poème? En bref, la traduction menacerait de prononcer le divorce de la forme et du fond que la poésie avait harmonieusement réunis et c'est ce que signale 
Baudelaire lorsqu'il déplore l' "affreuse imperfection» du "moulage de la prose appliqué à la poésie » et du "mal » que représente, au contraire, la « singerie rimée ${ }^{5}$ ». Le $\mathrm{XX}^{\mathrm{e}}$ siècle mit fin à cette hésitation et trancha en faveur de l'abolition de la rime. La multiplication des traductions universitaires entraîna, semble-t-il, la prévalence du sens sur la forme, tant et si bien que les poètes eux-mêmes ne se risquèrent plus à traduire les rimes. Valéry et Bonnefoy, l'abandonnèrent notamment pour leurs traductions respectives de Hardy et Donne ${ }^{6}$. L'illustre angliciste français, Robert Ellrodt, dans un article de la revue Palimpsestes, confirme cet état de fait : «l'abandon de la rime me paraît acceptable, et même généralement souhaitable aujourd'hui ». L'auteur poursuit : «du moment que l'écho de la rime est absent, la longueur du vers peut varier ${ }^{7}$ ». La traduction procédant par effets de compensation successifs, il pourrait sembler curieux qu'une perte doive en entraîner une autre. Il n'en demeure pas moins que la traduction de la rime s'avère une entreprise périlleuse et c'est pourquoi j'ai immédiatement accepté de relever le défi que Simon Jardin me proposait lorsqu'il m'a demandé de traduire son recueil, Voies de disparition ${ }^{8}$, en vers réguliers rimés.

Quoique déjà rompue à la traduction romanesque, et ayant traduit quelques poèmes en vers libres, je pressentais que la tâche serait difficile. Si l'attention particulière portée à la rime et aux effets formels représentaient une nouveauté, la manière de travailler comprenait également sa part d'inédit. Alors que je m'étais auparavant consacrée à des auteurs défunts je jouissais, pour la première fois, de la possibilité d'un dialogue avec un auteur bien vivant. Les traductions que nous commenterons dans cet article résultent ainsi du fruit de constants allers-retours entre l'auteur et le traducteur. Ces échanges répétés m'ont été particulièrement utiles: je pouvais adresser au poète la plus infime de mes interrogations concernant le sens d'une image ou l'importance d'une allitération. Cette démarche nous pousse aujourd'hui à écrire à deux - et à briser l'idée quelque fois trop répandue selon laquelle l'auteur ne serait pas le mieux placé pour aider son traducteur.

4 Nous tâcherons de montrer la manière dont la transposition des règles formelles contrevient aux normes de l'édition contemporaine et tend à faire de la traduction de la poésie une activité marginale, sinon illicite.

5 Simon Jardin évoquera, en premier lieu, la manière dont le poète peut s'assimiler au hors-la-loi dans la mesure où ces deux figures se voient accusées d'emprunter une mauvaise voie. Je proposerai ensuite, en écho à la thèse de Simon Jardin, une analyse des différents crimes dont on inculpe la traduction de la poésie. Nous illustrerons ensuite notre propos par une étude générale des dix poèmes traduits ainsi que les commentaires plus spécifiques des textes « Eye Liner » et « Ne plus se pardonner.»

\title{
Écrire en vers, écrire en marge
}

\author{
ÇA c'est naïvement une impudente pose ; \\ C'est, ou ce n'est pas ça : rien ou quelque chose... \\ $[\ldots]$ \\ C'est un coup de raccroc, juste ou faux, par hasard... \\ L'Art ne me connait pas. Je ne connais pas l'Art'.
}

6 C'est par ces vers de Tristan Corbière que je veux débuter mon propos. Ils présentent l'avantage d'éclairer la difficulté de ce qui m'attend, à savoir produire un discours sur ce fameux « ÇA », ma poésie donc, tout en nous plongeant immédiatement au cœur de 
l'exclusion qui caractérise la vocation de poète. En s'attardant sur le dernier vers, on peut relever la manière dont le retournement ironique de la formule en guise de défi, donne lieu à une sorte de chiasme par lequel le poète se retrouve enfermé dans cet art dont il se sent l'étranger. Il semble ainsi qu'on entre en poésie comme par effraction, et que le poète ne s'y sent jamais tout à fait chez lui.

Intrus en poésie, le poète l'est encore en société. Lorsqu'il entre dans un café, une administration, un appartement, la question ne tarde pas à fuser : « et toi, qu'est-ce que tu fais dans la vie? ", ce à quoi le poète, sûr de son effet, répond : "de la poésie ", ce à quoi l'autre personne, vraisemblablement insatisfaite et déjà passablement agacée : « non mais qu'est-ce que tu fais vraiment dans la vie? ». L'histoire en vient à se répéter et l'existence, à rimer. Mais ce redoublement de la question qui structure mon quotidien, est d'autant plus frappant qu'il reproduit le fameux procès de Joseph Brodsky en $1964^{10}$ : l'interlocuteur prend le visage du juge, et le poète, celle de l'accusé. La mise en question se change en mise en accusation. C'est d'abord ainsi que le poète se trouve hors la loi. Frappé dès l'origine d'étrangeté et d'infamie, le cheminement du poète apparaît soit comme une impasse, soit comme une mauvaise voie. Si la poésie est une faute, c'est qu'elle ne constitue pas une voie d'insertion, mais une voie de disparition.

Le titre de mon livre ironise sur cette idée. Ce recueil de poésie paru aux éditions du Cerf en 2019 réunit des poèmes écrits entre 2012 et 2018. Faisant suite à un deuil, il évoque le chaos d'une époque où l'on ne croit plus en rien. De poème en poème, il met le lecteur face à l'idée d'une mort programmée. Il s'agit de s'emparer aussi bien de sujets contemporains qu'intemporels en alliant forme classique et syntaxe moderne. Son pari consiste à confronter le vers régulier et la réalité de notre monde.

9 La mauvaise voie de la vocation de poète se double de la mauvaise voie du vers régulier. À l'exclusion de la cité et du marché, s'ajoute l'exclusion de l'édition de la poésie. Car l'élaboration d'une poésie versifiée se heurte à la norme éditoriale actuelle. Mon propos est d'étudier comment en poésie la règle est devenue hors la loi.

Le rejet de la règle de la poésie contemporaine se fonde sur l'idée que celle-ci renverrait à un ordre culturel, social, politique contraignant et excluant: le respect de la règle prolongerait cet ordre au sein du langage et de la littérature. La liquidation de la règle permettrait la liquidation de cet ordre.

11 C'est précisément ce que je récuse : cette analyse décrit à la limite la situation poétique $\mathrm{du}$ début $\mathrm{du}$ dix-neuvième siècle, la règle renvoyée au classicisme se rapportant à l'Ancien Régime. Cependant, la chute de l'Ancien Régime a renouvelé l'exclusion platonicienne : le poète ne s'insérant pas au processus productif, perd sa place dans la nouvelle société capitaliste de la révolution industrielle. Il semble perçu comme une survivance vouée à disparaître : ce qui était la voie royale devient la mauvaise voie. Cette condition d'exclu va néanmoins susciter une lignée de poètes dont la figure sera celle du fou avec Nerval, du blasphémateur avec Baudelaire, du maudit avec Verlaine, du révolutionnaire avec Hugo et du rebelle avec Rimbaud, et ce jusqu'au milieu du vingtième siècle avec les points culminants de la Résistance et de la négritude. Cette lignée rassemble des auteurs qui maîtrisant la règle, jouent à la subvertir.

Cette singularité culmine lorsque la figure du poète et celle du hors la loi convergent au travers d'auteurs comme Villon, Lacenaire ou Genet : respect de la règle et défiance de la loi s'y affirment sans contradiction. La «Ballade des pendus » est ainsi élaborée à 
partir du décasyllabe. Elle reprend la forme de la ballade avec son refrain et son envoi. L'ouverture du poème est d'autant plus marquante qu'elle place dans la bouche des condamnés, un appel à l'humanité future : « Frères humains qui après nous vivez ${ }^{11}$ ».

Or cet appel des pendus se confond avec celui des poètes, dès lors que ceux-ci prétendent s'adresser à des êtres qui ne sont pas encore venus à l'existence. Les pendus et les poètes se tournent vers l'avenir à la recherche d'un autre jugement. Cet espoir et cette exigence se retrouvent dans un quatrain de Jean Genet. L'alexandrin porte la révolte du poète qui interroge la condamnation d'un amant.

Nous n'avions pas fini de nous parler d'amour.

Nous n'avions pas fini de fumer nos gitanes.

On peut se demander pourquoi les cours condamnent

Un assassin si beau qu'il fait pâlir le jour ${ }^{12}$.

La tonalité élégiaque des deux premiers vers débouche sur la remise en cause du jugement rendu par les cours : il oppose à la justice des hommes, une autre valeur : la beauté. La poésie apparaît ainsi comme la seule langue qui puisse prétendre faire face à la condamnation, parce qu'elle est la seule langue qui puisse faire face à la mort. C'est cette singularité qui fait porter à l'alexandrin les derniers mots de Lacenaire avant son exécution. Poèmes et lois se font face, lesquels relirons-nous?

Que voulez-vous de moi ? vous parlez d'échafaud?

Me voici... j'ai vécu... J'attendais le bourreau ${ }^{13}$.

Le vers apparaît en dernière instance comme la marque d'un langage d'exception réunissant le poète et le criminel : entre langage secret, code d'honneur et goût du cachet. Hors la loi, le poète le sera du moment qu'il fera montre d'une langue exceptionnelle. La règle en poésie n'est pas un rappel à la loi.

Le rejet de la règle désigne en vérité une injonction à rentrer dans le rang. Refuser au poète une langue exceptionnelle, c'est le banaliser; le banaliser, c'est le réduire à néant. Si la règle constitue l'ensemble des règles de versification et de rhétorique, qui rendent possible un discours, désinvestir ces moyens conduit à perdre la possibilité de nommer le monde. C'est ce qu'on observe dans la deuxième partie du vingtième siècle. Les poètes considérés comme majeurs se détournent radicalement de leur temps et du même coup des moyens de le dire. Ce désinvestissement s'accompagne d'une dissémination progressive de la poésie. La poésie sortant d'elle-même est désormais partout : chanson, roman, et cinéma; elle est dans la rue et dans le métro, dans la conversation et dans la politique: partout, du moment qu'elle n'est plus le fait d'un poète. Les auteurs sont à leur tour invités à faire sortir leur poésie d'elle-même : la poésie sera graphique, sonore, ou performance. Bière sans alcool, coca sans sucre, poésie sans langage : elle ne fera pas exception.

17 Cette banalisation se prolonge dans l'usage que fait la publicité des moyens jadis dévolus à la poésie : ceux-ci deviennent à partir des cinquante le monopole de la communication publicitaire. Les slogans sont en effet construits sur des hémistiches ou des octosyllabes avec rimes internes: Champomy: "Sans alcool la fête est plus folle » ou BMW : «Le plaisir de conduire ». Celui de l'assurance MMA mérite qu'on s'y attarde : "Zéro tracas zéro blabla MMA », il associe rimes parallélisme et répétition anaphorique ; l'apposition finale du nom de la marque met en valeur un acronyme et une réduction du nombre de syllabes (4/4/3) qui évoquent la simplification que le slogan cherche à promouvoir. Les publicitaires empruntent l'ensemble des figures de style : paronomase (GMF : "Assurément humain »), oxymore (Gini : «La plus chaude 
des boissons froides»), périphrase (Kiri : "Le fromage des gastronomes en culottes courtes ») parallélisme ("Carglass: Carglass répare, Carglass remplace »), ou encore zeugma (Monnaie de Paris : «Frappe la monnaie et les esprits »). Ces procédés de mise en valeur sont utilisés désormais à des fins commerciales : susciter le désir, atteindre et fédérer les publics-cibles, vendre les produits de la société de consommation. Tout entière dirigée vers son efficace, la publicité réussit à informer notre vision du monde d'une manière plus profonde que la poésie. Et pour ce faire : les publicitaires usent des procédés que les poètes refusent. Cette banalisation désigne une expropriation.

Ma démarche consiste en une réappropriation des moyens et des procédés confisqués par la publicité. Ce n'est donc pas hors d'elle-même, mais en elle-même que la poésie peut et doit trouver les armes pour affronter le monde du nouveau millénaire. L'enjeu de cet affrontement est la possibilité de donner à notre monde un autre sens que celui qu'il produit sur lui-même. Cette mission de la poésie paraît d'autant plus décisive que les sociétés néo-libérales dans lesquelles nous vivons, sont riches en bien, et pauvres en sens. La marginalité du poète le place ainsi directement face à ce monde. Il s'agit donc d'assumer, plutôt que de déplorer, l'exclusion qui le frappe. Mon projet poétique, entre sursis et survie, peut donc s'énoncer ainsi : proposer à la place d'un discours qui nous achète, une poésie qui nous achève.

Simon Jardin

\section{Traduire en anglais, traduire en justice}

Si la publicité paraît s'être emparée des règles de l'écriture poétique, elle poursuit son opération de main-basse sur le domaine littéraire en semblant dicter les normes du marché éditorial contemporain. L'article « Traduttore, traditore » dont le titre reprend le célèbre adage italien selon lequel tout traducteur serait un traître, paru dans la revue Les Cahiers de la publicité en 1967, le montre bien. L'auteur y livre des conseils afin de traduire les campagnes publicitaires étrangères et se fonde, pour ce faire, sur l'antagonisme qui opposerait traducteur publicitaire et traducteur littéraire.

La traduction littéraire atteint sa plus grande maîtrise par l'effacement de la personnalité du traducteur au profit exclusif de celle de l'auteur. Il ne s'agit pas de faire passer pour française une œuvre étrangère, mais au contraire de rendre avec la plus grande vérité possible son caractère étranger et original. Le traducteur voudrait devenir cet étranger qui parlerait notre langue ${ }^{14}$.

21 Cette conclusion a de quoi surprendre le littéraire qui la découvre : l'éloge qu'elle brosse de l'étranger préfigure singulièrement la traductologie de la seconde moitié du $\mathrm{XX}^{\mathrm{e}}$ siècle - qui eût cru qu'un publicitaire se ferait, en avance, le chantre de la traduction comme épreuve de l'étranger qu'Antoine Berman ne loua qu'à partir des années $1990^{15}$ ? Mais le publicitaire surenchérit :

Le traducteur publicitaire doit au contraire, sur un canevas étranger faire une œuvre française. Traduire de façon séduisante, crédible, vivante, convaincante, bien adaptée à la mentalité de ses lecteurs, un modèle conçu par et pour des étrangers ${ }^{16}$.

Propos dont tout traducteur littéraire a fait l'expérience amère, tâchant de défendre son projet dans le bureau d'un éditeur peu convaincu. La traduction publicitaire telle qu'elle se pratiquait à la fin des années 1960, c'est-à-dire la traduction qui vise à gommer les aspérités et qui privilégie la culture cible à la culture source, établit ainsi les normes de la traduction littéraire telle qu'elle se déploie aujourd'hui. En ce sens, 
l'expropriation qu'évoquait Simon Jardin tout à l'heure se redouble au moment de la traduction, et toute traduction qui se fixe pour but de restituer quelque chose des règles formelles de l'original est accusée, à l'image du poète, de faire fausse route. Le traducteur littéral, comme le poète, s'apparente à la figure du vagabond - quoique son errance ne se limite plus à celle qui le mène aux marges de la société marchande puisqu'elle le mène à vadrouiller entre les langues.

Cet exil est précisément ce que les éditeurs contemporains cherchent à cacher lorsqu'ils demandent à leurs traducteurs de se ranger du côté de leur langue de rédaction et qu'ils font prévaloir les lois de la grammaire à celles de l'auteur. Ce qu'on exige du traducteur, ce n'est pas tant qu'il maîtrise sa langue, qu'il ne se laisse maîtriser par elle. Ainsi, André Markowicz a dû batailler pour imposer ses traductions de Dostoïevski, qu'il estimait jusqu'alors englué dans un français balzacien ${ }^{17}$. Il ne s'agit pas ici de juger de la qualité du travail du traducteur - sa démarche évoquant un effet de mise en abyme lorsqu'on sait que la première œuvre de Dostoïevski fut la traduction d'Eugénie Grandet ${ }^{18}$ - mais de mieux cerner les critères des maisons d'édition. Si la traduction de Markowicz choquait autant, c'était parce qu'elle ne craignait pas de restituer, notamment, les nombreuses répétitions du romancier russe. Or, la répétition souffre, en France, de son statut de parent pauvre de la figure de style. Que Dostoïevski, ce maître incomparable, puisse y recourir, demeurait, pour certains, impensable. Les éditeurs contemporains ressemblent en cela à leurs prédécesseurs au XVIII ${ }^{\mathrm{e}}$ siècle, qui s'offusquaient de ce que les héros homériques puissent, après le combat, prosaïquement passer à table ${ }^{19}$. Les règles de l'édition en matière de traduction tendent ainsi à produire ainsi des textes standardisés, dont la langue servile peut difficilement être qualifiée de littéraire.

Le refus de céder à ces pratiques s'apparente dès lors, sinon à une transgression, du moins à une révolte. C'est ce que souligne le chercheur Lawrence Venuti lorsqu'il évoque la traduction littérale, qu'il qualifie de " dissident cultural practice ${ }^{20}$ » Selon lui, cette pratique relève du refus de " the dominant aesthetic in the receiving situation ${ }^{21}$ ". Ainsi, la traduction en anglais d'un sonnet de Simon Jardin relève d'un double défi : il s'agit non seulement d'imposer un modèle devenu mineur - la poésie anglosaxonne contemporaine recourt davantage à la prose ou aux haïkus qu'à cette forme traditionnelle - mais également de l'imposer dans sa forme française, qui diffère du schéma anglais habituel. Nous pourrions toutefois nous interroger sur le statut hétérodoxe d'une traduction vers l'anglais, langue dominante par excellence, dont l'emprise s'exerce à travers le monde des affaires et de la culture populaire. La traduction, loin de paraître le fruit d'une rébellion, semblerait davantage relever de l'annexion d'une culture minoritaire par une culture hégémonique. Afin de contourner cet écueil, Venuti préconise de devenir « un nomade dans sa propre langue, l'anglais, un fugitif au sein de sa langue maternelle ${ }^{22} »$. En bref, il s'agit d'afficher l'exil pour éviter de mettre le texte original en péril.

Mais les accointances du traducteur et du hors-la-loi ne se cantonnent pas à la figure du vagabond et de l'exilé. L'une des métaphores les plus anciennes qui caractérise la traduction est celle du traducteur-pilleur qui enrichit sa propre langue grâce à la traduction ou à l'imitation des langues anciennes ou étrangères. Dans sa conclusion de la Défense et illustration de la langue française, Du Bellay conseille ainsi aux Français, afin d'accroître leur langue, de "[marcher] courageusement vers cette superbe cité romaine : et des serves dépouilles d'elle, [orner] vos temples et autel ${ }^{23} »$ ou encore de 
"[piller], sans conscience, les sacrés trésors de ce Temple delphique ${ }^{24}$ ». La langue vulgaire s'enrichit ainsi du butin que le poète, ici traducteur et pilleur, rapporte de la mise à sac des langues mortes. Valéry Larbaud, traducteur de Joyce et poète, développe également l'analogie qui compare le traducteur au voleur. Dans son ouvrage consacré à la traduction, Sous l'invocation de Saint Jérôme, il assimile le plaisir du traducteur à celui de la transgression des lois de la propriété intellectuelle qu'implique son activité. Le traducteur ne serait qu'un plagiaire dont l'entreprise d'imitation serait socialement acceptée $^{25}$. L'image plus contemporaine, issue de la pensée des transferts culturels, du traducteur comme passeur, souligne à nouveau l'illégalité inhérente à l'activité de traduction. Le traducteur-passeur est en effet celui qui s'efforce d'importer un texte étranger là où il n'a pas droit de cité : on songe notamment aux imprimeurs de La Haye qui, au XVIII siècle, publiaient et exportaient en France des traductions fidèles d'auteurs étrangers qui seraient tombées sous le coup de la censure royale ${ }^{26}$. Le traducteur s'apparente dès lors au contrebandier, à celui qui fait traverser les frontières aux marchandises illégales.

Tantôt vol, escroquerie ou fraude, les métaphores qui soulignent la transgression des règles propres à la traduction s'aggravent pourtant et la font passer d'activité délictuelle à activité proprement criminelle. René Ladmiral, fervent défenseur de la traduction cibliste, c'est-à-dire de celle qui privilégie le fond à la forme, accuse en effet ses adversaires, les sourciers, d'être les auteurs de viols contre leur langue maternelle.

Alors que les ciblistes se veulent éminemment respectueux du plaisir des langues, du plaisir propre à la langue dans laquelle on parle (ou écrit), c'est-à-dire en l'occurrence qu'ils entendent respecter la langue-cible, je suis tenté de dire que la logique des sourciers, c'est la logique du viol ${ }^{27}$ !

Les sourciers, en conférant des accents étrangers à leur langue-cible, outrepasseraient ainsi le consentement de cette dernière. "Non, c'est non!» paraît dire Ladmiral, semblant curieusement ignorer que l'image à laquelle il recourt implique d'envisager la langue comme un corps, ce qui le placerait, paradoxalement, davantage du côté de la lettre que de l'esprit. Mais, et pour en revenir à ce qui semble être la véritable pensée de l'auteur, lorsque Ladmiral, reproche aux sourciers de se comporter comme des violeurs, il ne les accuse pas seulement de malmener leur langue cible, mais bien de frayer avec l'ennemi. En effet, le traductologue semble souligner le danger qu'il y aurait à trop s'acoquiner avecle texte-source. Ce dernier aurait, sur le traducteur, une influence pernicieuse, pensée qui paraît dominer l'enseignement de la traduction littéraire.

La traduction y est en effet souvent présentée en termes herméneutiques et les ouvrages de référence demeurent bien ceux qui assimilent cette pratique à l'interprétation ${ }^{28}$. Dès lors, la traduction ne peut plus se cantonner à l'activité qui fait passer un texte d'une langue à l'autre, mais se doit également de comprendre un pan critique. C'est l'idée selon laquelle le traducteur est le premier critique de l'œuvre qu'il traduit. On attend alors de l'élève traducteur qu'il affronte son texte à l'aide d'un appareil critique solide, dont les principaux éléments demeurent le structuralisme et la théorie de la réception, deux courants qui conduisent à l'effacement des prérogatives de l'auteur, qu'elles fassent de l'œuvre littéraire un réseau de significations ou qu'elles le soumettent aux attentes du lecteur ${ }^{29}$. Le bon élève traducteur doit ainsi maintenir une supposée neutralité scientifique face à l'auteur, mauvaise fréquentation par excellence, tout en s'efforçant de flatter l'horizon d'attente du lecteur. Ces recommandations, que j'applique scrupuleusement lorsque je me consacre à la 
préparation de ma thèse de littérature britannique, me paraissent toutefois bien peu recommandables dans mon travail de traduction. Il me semble, à titre personnel, que la traduction procède par affinités électives. En effet, si les traducteurs choisissent de s'emparer de textes qu'ils n'ont, en réalité, nul besoin de traduire puisqu'ils en connaissent la langue d'origine, c'est bien qu'ils y éprouvent un certain plaisir qui, à mon sens, n'est pas seulement celui de la transmission, mais bel et bien celui de la réappropriation d'un texte. Or, à moins que l'on puisse établir que tout traducteur soit nécessairement soumis à une forme de pulsion masochiste, ce plaisir me paraît devoir reposer sur l'adhésion à la pensée de l'auteur.

Ainsi, le traducteur qui aspirerait à restituer les effets formels et la pensée de l'auteur se voit condamné à vagabonder à la marge du marché de l'édition, accusé de crimes par les penseurs de la traduction et soupçonné de mauvaises fréquentations au sein des institutions universitaires.

\section{Amélie Derome}

\section{Voies de disparition}

Les traductions qui vous sont présentées aujourd'hui, prennent source dans le désir de mettre en image des extraits de Voies de disparition. Réalisant des films depuis six ans en parallèle de l'écriture, j'ai voulu que la parution du recueil soit accompagnée d'une série de films mettant chacun en scène un poème. Ce projet a pour titre Nous resterons inconsolés ${ }^{30}$. Alors que nous recherchions les canaux de diffusion adaptés à un tel projet, la question du sous-titrage s'est très vite posée. Nous avons fait le choix d'élaborer des traductions littéraires, plutôt que littérales. La genèse de ces traductions constitue ainsi une double rupture sur les pratiques conventionnelles. D'une part, il s'agit de substituer à la lecture de poésie l'intensité du cinéma ; d'autre part, il s'agit de conférer aux sous-titres une valeur en soi. Dévoiler cette genèse est nécessaire dans la mesure où c'est de celle-ci que résulte le choix de poèmes. Le projet cinématographique s'appuie sur un dispositif de mise en scène simple : des acteurs affrontent la caméra en position de condamné à mort. Tenus en joue, en l'attente d'une exécution, ils déclament les poèmes, comme des dernières volontés. Il fallait choisir des poèmes qui, prenant la forme de profession de foi, ne rentrait pas en contradiction avec la situation. Les acteurs étant masqués par un bandeau, les thèmes de la vision et de l'aveuglement ont également été privilégiés.

Les poèmes sont tirés de différents moments de mon livre qui se divise en cinq parties : « Ne plus se pardonner ${ }^{31}$ » ouvre à la fois le livre et la série de film. «Preuve ${ }^{32}$ » est tiré de la première partie, Régression à l'infini, qui évoque l'absence de fondement, la rencontre avec l'infini et la mise au jour d'une origine innommable. Aucun poème n'est tiré de la deuxième partie, Chambres noires, qui explore l'enfance, l'enfermement, les spectres du cinéma. "Eye Liner ${ }^{33}$ " et "Incrustation ${ }^{34}$ ", proviennent de la partie centrale, Jeu de survie, qui décrit la rencontre manquée avec le réel, entre monde fantôme et fantasme virtuel. Les trajectoires hasardeuses et les chemins qui ne mènent nulle part traversent la quatrième partie : Balles perdues, d'où sont tirés « Promesse ${ }^{35}$ ", « Presque rien ${ }^{36}$ » et « Somnolence ${ }^{37}$ ». La dernière partie, Procession, se confronte à la mort et au deuil sur le plan individuel comme dans « Saison ${ }^{38}$ » et " Trêve ${ }^{39}$ » et sur le plan collectif, comme dans « Inconsolés ${ }^{40}$ ». 
33 l'image du livre pour la construction duquel j'ai gardé à l'esprit les principes esthétiques de l'unitas et de la varietas. C'est de cette unité dont il me faut vous parler maintenant: la traduction des textes a exigé de dégager la manière dont je me suis approprié les règles de la poésie.

Cest l'expression de notre monde et des émotions qui lui sont propres qui dirige mon emploi du vers. Les choix et les contraintes rendent à mes yeux possibles cette expression. Mon travail sur le vers régulier se fonde sur l'emploi d'une syntaxe ramassée et le rejet des archaïsmes: j'évite dès lors l'excès d'anaphores, l'excès d'inversions, et surtout la diérèse. Je prends aussi le risque du prosaïsme, à la suite de mon expérience de lecteur: en effet, même les tragédies ne vont pas sans quelques prosaïsmes. C'est souvent une part qu'on néglige, elle me parait importante à relever. Une telle idée s'inscrit plus généralement dans une alternance entre éléments abstraits et éléments concrets qu'élaborent mes poèmes. L'emploi de champs lexicaux issus de domaines techniques de notre temps, par exemple : le jeu vidéo ou le cinéma, structure certains de mes textes. Faire pénétrer ces mots et ces expressions dans l'espace du vers constitue à la fois un plaisir pour le poète, et une nécessité pour la littérature. Dans cette perspective, mon vers se distingue par le non-respect du rejet de l'hiatus, qui me semble empêcher l'intégration de nouveaux champs lexicaux (une expression comme «métro aérien » par exemple ne peut pas prétendre à exister poétiquement). et féminines, la césure et la fameuse règle des «e » muets. C'est le cas de tous les poèmes qui vous sont présentés à l'exception de «Preuve" qui est composé en vers libres non rimés. Je porte une attention particulière aux choix de la strophe : j'emploie majoritairement des strophes isométriques (le même mètre, la même strophe). Les strophes carrées (lorsque le nombre de syllabes est égal au nombre de vers) me semblent adaptées des poèmes où le moi s'affirme, puisqu'il apporte une stabilité et un enfermement particulièrement évocateurs comme dans « Somnolence » et « Trêve ». La strophe verticale et son accélération caractéristiques dynamisent le propos comme dans « Inconsolés ». De manière générale, il s'agit de jouer des effets d'équilibres et de déséquilibres, en mêlant le pair et l'impair comme dans «Incrustation » avec trois strophes de quatre vers ou « Ne plus se pardonner » avec trois strophes de six vers.

Mais travailler sur le vers exige une attention accrue à l'accentuation et la découpe. Les mètres déterminent des accents principaux en fin de vers ou d'hémistiche. Déplacer les accents induit des effets de sens et de sons: les rejets et les contre-rejets sont construits à partir de la mise en valeur de l'accent final et de la dernière syllabe du vers à l'instar du vers 9 de « Presque rien » et du vers 9 de « Trêve ». L'attention aux accents permet d'imprimer un rythme émotionnel au vers : que ce soit la lancinance frappante à la fin d' "Eye Liner " ou dans le poème "Somnolence ", ou la nervosité perceptible dans "Trêve ", "Presque rien » ou " Promesse ». Paradoxalement il me semble qu'un vers lancinant exige plus de heurts et de décalages, qu'un vers nerveux qui peut être obtenu au moyen d'un parallélisme épousant les vers ou les hémistiches. Ce sont deux rythmes dont ma poésie cherche à opérer la réunion; entre lancinance et nervosité, la poésie du sursis est aussi une poésie du sursaut.

La musicalité que donne le vers régulier rimé, n'est dès lors pas conçue pour adoucir, mais pour asséner : le recours aux répétitions («Inconsolés » v. 4 et v. 10, «Trêve » v. 12, Preuve premier et dernier vers de chaque strophe), aux accumulations 
(«Incrustation » v. 7, «Trêve » v. 8), aux rimes internes («Promesse » v. 5 et v. 6, «Inconsolés» v. 3), aux paronomases («Saisons » v. 1, v. 5, v. 9), aux assonances ("Presque rien » v. 6 et v. 9-11) et aux allitérations («Incrustation » v. 7, "Presque rien » v.14), cherche à atteindre le lecteur, assez pour le mettre face au monde qui l'entoure.

38 C'est à partir de ces quelques éléments d'analyse que la traduction a pu débuter. L'orientation générale que j'ai proposée alors à Amélie Derome, consistait à assumer le péril qu'implique la traduction de la poésie. Il fallait tenter de restituer les vers, les rimes, la musicalité : la forme interpénétrée au fond autant que possible. Il me semble c'est à ce prix que la poésie peut se transmettre. Ce désir d'être traduit prend origine dans les traductions qui m'ont permis de découvrir nombre d'auteurs comme Mandelstam ${ }^{41}$ ou Rilke ${ }^{42}$, Brodsky ${ }^{43}$ ou Markish ${ }^{44}$, Pessoa ${ }^{45}$ ou Akhmatova ${ }^{46}$, Tsvetaieva ${ }^{47}$ ou Celan ${ }^{48}$, qui hantent et influencent mon écriture. Une poésie qui prétend affronter le monde, se doit d'être capable d'affronter une autre langue, en particulier la langue dominante par excellence : l'anglais.

Simon Jardin

\section{Voies de transmission}

Affronter le péril de la traduction en m'efforçant d'interpénétrer le fond et la forme : voilà qui ne relevait pas d'une mince affaire. Enfin, puisqu'il fallait bien commencer, je le fis en décidant des équivalences à établir pour la traduction des vers. Pour l'alexandrin, vers du sonnet et de la tragédie, j'optai pour le pentamètre iambique, vers noble et shakespearien par excellence. Quant aux octosyllabes, je décidai de les traduire par des tétramètres, tantôt iambiques tantôt trochaïques, dont la construction anglaise s'apparente à celle que l'on trouve en français. Ce choix d'équivalence a orienté la manière dont j'ai entamé ma traduction de l'ensemble des dix textes de Simon Jardin dont il est question dans cet article. Je commençais en effet par la restitution des octosyllabes puisqu'elle nécessitait moins d'aménagements que la traduction des alexandrins.

41 Par chance, le titre du projet de court-métrages reprenait le premier vers d'un poème en octosyllabes, « Nous resterons inconsolés ». La manière dont j'ai traduit ce texte me paraît avoir influencé l'ensemble de ma démarche.

J'ai en effet proposé deux versions distinctes, dont les différences reposaient sur la façon de traduire inconsolés. Dans le premier, inconsolé donnait disconsolate, mot composé de deux iambes et qui permettait de traduire ce vers qui revient tout au long du poème comme un refrain par un iambe : "We shall remain disconsolate ". Le suffixe latin de disconsolate, ainsi que la syntaxe naturelle du vers, donnaient une tournure moderne à cette première version anglaise. La seconde se fondait sur la traduction d'inconsolé par unconsoled, syllabe accentuée suivie d'un iambe. La traduction iambique s'est avérée plus délicate, et j'ai dû recourir à la construction do + infinitif, qui produit un effet de renforcement, afin de retrouver le rythme souhaité, ce qui donnait : « For we do tarry unconsoled ». Or, la combinaison de cette construction verbale et du registre élevé du verbe to tarry (demeurer) conférait au vers une tonalité plus archaïque entièrement absente du poème original. Lorsque Simon Jardin a pris connaissance des deux possibilités, nous avons compris que la question de la traduction de sa syntaxe condensée ne pouvait être éludée, quoiqu'il ait, pour d'autres raisons, été sensible à la 
deuxième version du poème. Cette nouvelle règle ne me serait sans doute pas apparue avec autant de force si je n'avais eu l'opportunité de travailler main dans la main avec l'auteur.

Ce premier essai m'a également permis de saisir l'importance qu'il y avait à ne pas figer les rimes trop tôt, mais plutôt à conserver un panel de rimes possibles, jusqu'à la rédaction finale du poème traduit. J'ai ainsi compris qu'il me fallait commencer par repérer les mots clés du poème, c'est-à-dire ceux qu'il était impossible de traduire par une périphrase et qui orientaient le sens général du texte. En ce sens, la traduction d'un poème commence rarement par le premier vers, mais par ceux qui comprennent de tels mots clés. Je vais tâcher d'illustrer mon propos en expliquant brièvement ma traduction du premier quatrain de saison, que voici :

Un peu enfant, un peu fantôme

Au bord de ce siècle indécent,

J'ai lu l'avenir dans mes paumes

Et mes paumes étaient en sang ${ }^{49}$.

Ici, j'ai dû troquer les rimes croisées du poème saison pour des rimes embrassées. Il me fallait en effet commencer par la traduction du troisième vers, puisque l'original, chose rare, m'offrait la possibilité d'un mot-à-mot : «I read the future in my palms ». Pourtant, aucun mot anglais renvoyant au fantôme ne rimait avec palms. Le pluriel posait également un problème dans la mesure où le $[\mathrm{s}]$ se prononce en anglais. Je ne pouvais pas traduire paumes au singulier puisque le quatrième vers renvoie aux stigmates du Christ, qui sont bien au nombre de deux. De la même manière, il aurait été malheureux de démultiplier la personnalité le poète en le comparant à deux fantômes. La solution que j'ai trouvée fut donc de faire rimer les deuxième et troisième vers en privilégiant la traduction suivante "In this age of little qualms ». Il ne restait plus qu'à faire rimer le quatrième et le premier vers, ce qui advint grâce à l'inversion de l'ordre du fantôme et de l'enfant. Le quatrain complet donne ainsi :

Quite the ghost and quite the bud

In this age of little qualms,

I read the future in my palms

And saw that they were drenched in blood.

Après m'être attelée à la traduction des poèmes en octosyllabes, je m'attaquais à ceux qui étaient rédigés en alexandrins. La traduction de ces derniers s'est avérée délicate, puisque la souplesse du pentamètre implique, paradoxalement, davantage de maîtrise. Les difficultés de traduction de la rime demeuraient pourtant similaires, comme le montre le troisième quatrain de « Promesse ».

A mon tour de sentir et de lécher la plaie

De tout ce qui sera. Qui d'autre le pourrait?

Nul être de ce monde ici ne me relaie.

Je sens sur moi se refermer ses bords secrets ${ }^{50}$.

Ici, pour traduire la rime en "laie ", il me fallait commencer par traduire plaie, qui comprend un plus petit nombre de traductions possibles que "relaie ». Ma première version traduisait ainsi ce vers: "My turn has come to smell and lick the wound ». Je m'aperçus hélas qu'il n'existait aucune rime pour wound. Je trouvais pourtant le terme de womb, qui renvoie au ventre maternel et qui permettait une rime imparfaite. En brodant quelque peu la signification du vers "Nul être de ce monde ici ne me relaie", je finis par traduire : "No being traced my exit from the womb ", c'est-à-dire, nul être ne m'a suivi hors du ventre de ma mère. Personnellement très satisfaite de ma traduction, 
tout en demeurant consciente d'avoir légèrement outrepassé mes droits, je la soumis à Simon qui, naturellement, me rappela que le sens s'éloignait trop de l'original, quoiqu'il eût la gentillesse de me conseiller de conserver ce vers dans l'éventualité où je voudrais l'utiliser un jour. Afin de revenir dans le giron de la loi que m'imposait l'auteur, je me mis donc en quête d'un synonyme de wound et choisis cut, qui désigne la coupure et pus traduire : "My turn has come to smell and lick the cut ». Toutefois, je ne trouvais toujours pas d'équivalent anglaise de relaie qui rime en « ut » et dus réaménager le vers ainsi : « Beings in this world find my path shut » qui donne en français « Ma voie est fermée aux êtres de ce monde ", et qui suit davantage le sens original.

Si j'ai divisé mon travail entre poèmes en octosyllabes et poèmes en alexandrins, deux poèmes ont été travaillés entièrement à part, « Somnolence » et " Preuve », deux textes aux formes singulières. Voici le premier, composé de sept vers en heptasyllabes,

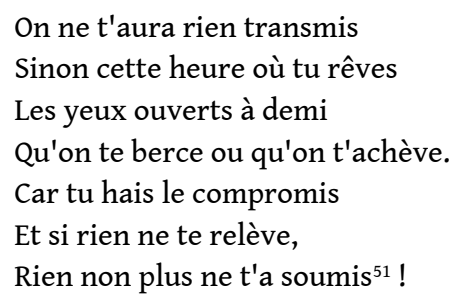

J'ai en effet longuement hésité sur le vers à choisir. Dans la mesure où le texte original évoque la berceuse, j'ai pensé opter pour le tetramètre anapestique (quatre fois deux syllabes non accentuées suivies d'une syllabe accentuée) vers privilégié de la poésie enfantine et des limericks. Ce choix donnait pourtant un tour comique au poème, entièrement exempt de l'original. Je me résignais donc à traduire l'heptamètre un tétramètre iambique, ce qui donnait ceci :

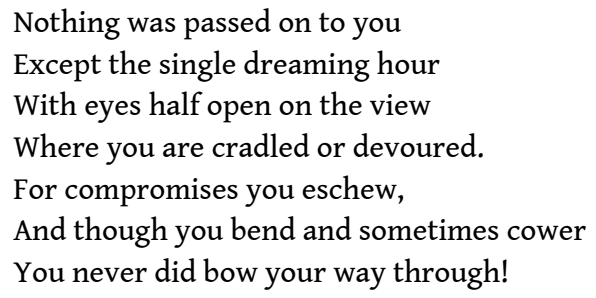

Lors de la relecture, Simon Jardin a très justement regretté ce qui s'est avéré un aveu d'échec de ma part, d'autant que ma traduction de la rime était imparfaite au quatrième vers. J'ai donc essayé de traduire par des trimètres trochaïques, mais la petitesse du vers s'est avérée insuffisante pour contenir le sens de l'original. Je fus, ensuite, frappée d'une inspiration arithmétique et crus pouvoir retrouver le chiffre sept en alternant trimètres trochaïques et tétramètres iambiques : $3+4=7$, me disais-je. Hélas, cet enchaînement faisait tomber le dernier vers à plat, alors qu'il sonnait comme une pointe dans le texte original.

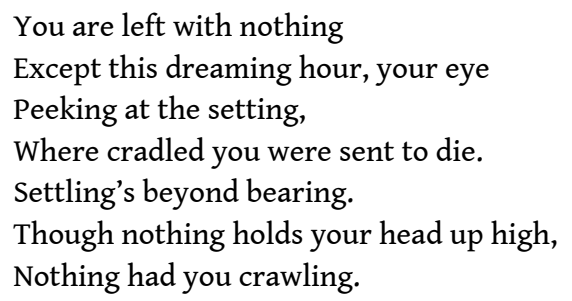


ernière instance, la piste qui a semblé le plus satisfaisante était celle qui consistait à alterner trimètres trochaïques et iambiques, ce qui soulignait bien la spécificité de la forme de l'original sans pour autant conserver la rengaine du chiffre 7.

You were left with nothing

But for this single hour

Eyes half open dreaming

They lull you yet then lower.

Bargain's not your thing

And though you tend to cower,

Nothing set you crawling!

51 Ce poème relevant d'une forme non-canonique, s'est ainsi paradoxalement révélé l'un des plus difficiles à traduire. A l'inverse, le poème "Preuve", présentant une contrainte apparemment très élevée, s'est avéré plus aisé. Ce texte met en effet en scène son propre effacement de strophe en strophe ${ }^{52}$. Un point a été particulièrement sensible : celui qui m'obligeait à traduire rive d'abord par un verbe, puis par un nom commun. Or, en anglais, aucun vocable ne peut recouvrir à la fois le sens de berge et d'attacher. J'ai donc tâché de trouver un terme qui puisse faire écho à l'original sans le traduire littéralement, et ai opté pour anchor, qui désigne aussi bien l'ancre - nous demeurons ainsi dans le registre maritime - que l'action d'attacher.

Avant d'achever ce récit de la manière dont j'ai procédé pour traduire cette sélection de poèmes, je souhaiterais aborder une difficulté de traduction d'ordre général et deux cas particuliers.

Les énumérations, fondées sur l'accumulation de termes concrets au sens précis et aux sonorités similaires, ont constitué une difficulté à laquelle je ne m'attendais pas. En voici une extraite du poème "Trêve ${ }^{53}$ »: « Mes larmes, mes sueurs, mes salives, mes moelles », présentant des allitérations en [s], en [1] en [m], et rimant avec "étoiles ». Aucun des quatre termes ne rimant avec l'anglais «stars ", j'ai dû rajouter un terme à l'énumération, tout en essayant de préserver l'allitération en $[\mathrm{s}]$ et en $[\mathrm{m}]$, et en remplaçant l'allitération en [1] par la répétition des sonorités proches [عə] et [I], mais également $[æ]$ et [a] ce qui donnait «my tears, my spits, my marrows, sweats and scars. » Ce poème présentait une difficulté supplémentaire, cette fois-ci plus légère, liée au premier vers : «Les murs repeint en noir et le poing ajouré ». Il me fallait trouver une manière d'éviter la tournure "paint it black", irrémédiablement associée aux Rolling Stones, afin de ne pas susciter la naissance d'un hypotexte relativement inapproprié. J'ai donc opté pour la formule suivante : « With walls in black new paint and fist ajar ». Enfin, je ne résiste pas à l'idée de conclure par un cas particulier de surtraduction, où la traduction naturelle d'un vers a suscité un nouvel effet. Le vers « Vat'en porter seins nus dans Paris effondré » a ainsi donné « Bare breasted, go in ruins of Paris bear ", vers encadré par deux homonymes.

Il me semble avoir montré, à travers cette étude générale de mes traductions des poèmes retenus, que la traduction de la poésie en vers réguliers relève davantage de la ruse, du louvoiement, que de l'écriture. La pratique de la traduction paraît ainsi s'apparenter à la notion grecque de mètis, le savant mélange de fourberie et d'habileté qui tire régulièrement Ulysse hors de l'embarras. Le héros qui fait preuve de mètis, comme le traducteur, est contraint de se mettre dans la peau de l'autre. Suivre la loi de l'auteur revient ainsi, paradoxalement, à une opération de tromperie.

Amélie Derome 


\section{Etude de cas 1, "Eye liner » : traduire peut être une plaie}

EYE LINER

Tu me reviens : ni voix ni tendresse ni île (Nihilisme de mes sensations à vingt ans) N'auront pu altérer la conscience fragile Que je te porte à toi, mon inachèvement !

J'aime, manquant de tout, la beauté qui mutile Avec des yeux pareils à ce double tranchant Qui seul a su mêler ma lutte à l'inutile Au regret de ton corps, les gouttes de mon sang.

Sans doute il m'a fallu sur le temps de survivre Prendre le temps d'aller vers ce qui me délivre. D'entre les rêves, prise à ta paupière sans

Nulle consolation, je disperse et ressens La pure et dérisoire insolence de vivre A ta paupière : hélas déjà lourde de sens.

Simon Jardin

EYELINER

Recalling you: no voice, no warmth nor reef (Rethink of when my senses were not five) Could ever tear away my dim belief In you: the incompletion I contrive!

I wish and want for grace that causes grief With eyes likes double-edges that can strive To mix my strife with what was never chief, My blood with longing of your flesh alive.

I guess I took, on time meant to survive, Some more to seek delivery. And I've, Amid dreams, seized within your eyelid,

Solace lacking, sowed but not got rid of the inane impertinence to thrive Against your lid: already heavy loaded. Traduction d'Amélie Derome Je voudrais pour débuter cette étude de cas dégager quelques éléments d'analyse du poème, « Eye Liner ». C'est un poème qui m'est cher parce qu'il faisait déjà partie de la première version de mon recueil en 2013. Il s'agit d'un sonnet irrégulier en alexandrins, sa particularité tient au fait que le sonnet est construit sur trois rimes au lieu des cinq traditionnelles, ce qui a pour conséquence le maintien de la rime en [ã] tout au long du poème. Nous nous trouvons donc face à deux quatrains construits sur deux rimes croisées, alternant féminines et masculines, et deux tercets construits sur deux rimes, 
enchaînant une rime plate, avant le retour des rimes croisées à la fin du poème. Approchons le détail de ce poème d'une manière linéaire.

\section{Premier quatrain - vers 1 à 4}

Le vers 1 s'ouvre sur un constat: "tu me reviens »; le poète s'adresse à un amour disparu; l'expression implique en même temps l'idée que cet être est une charge qui incombe au poète : l'être aimé prend la figure du spectre. La suite du quatrain explicite cette réapparition.

La deuxième partie du vers nous met face à une trinité : "voix " / "tendresse " / " île", qui se présente comme ce qui aurait pu détourner le poète de cet amour. Elle décrit comme une ambiance de carte postale, la possibilité d'une autre intimité, ou de l'intimité d'une autre.

61 Mais le vers 2 neutralise immédiatement cette possibilité. La rime annexée "ni île »/ «nihilisme » forme l'écho d'un néant qui vient caractériser la jeunesse du poète. La rime annexée souligne cette idée puisqu'elle forme un calembour. C'est une figure plutôt prosaïque qui renvoie à la poésie du Moyen-Âge, on peut penser à Marot qui reprend beaucoup les formes médiévales ${ }^{54}$, mais on en trouve chez des Parnassiens, elle fait alors figure d'ornement. Ici, elle donne l'idée d'une jeunesse qui est une blague, une mauvaise blague. En même temps, il fallait compenser la lourdeur du calembour dans ce poème lyrique, c'est pourquoi j'ai construit le vers sans césure. Les parenthèses viennent clore cette jeunesse sur elle-même.

Le vers 3 montre que par-delà cette parenthèse se maintient l'image obsédante de l'être aimé. Cela prend forme d'une attention, d'une conscience. Le poète parle de "conscience fragile» comme si cette conscience était encore inassumée, et que l'attention était lointaine.

3 Le vers 4 comporte un retournement renforcé par l'enjambement : cet amour devient assumé par le poète. On est face à un amour inachevé : une passade qui demeure à l'état de regret. Et ce regret devient constitutif du poète : le jeu des pronoms personnels : « je », « te », « toi », « mon » vient indiquer que le poète prend sur lui la responsabilité de l'inachèvement : il la fait sienne.

4 Si l'on observe le détail du premier quatrain, on note immédiatement qu'il est composé tout entier d'une unique phrase. Mais il y a un mouvement d'assomption tout au long du quatrain avec le ton qui s'affirme dès lors que les vers 1 et 2 paraissent hésitants avec leur césure et leurs accents déplacés et que les vers 3 et 4 se stabilisent avec une césure nette et des unités de sens coïncidant avec les hémistiches, jusqu'au point d'exclamation final.

\section{Deuxième quatrain - vers 5 à 8}

Alors que ce quatrain est à nouveau composé d'une unique phrase, le mouvement d'assomption trouve son point culminant dans ces vers où le poète fait une profession de foi. Ce statement est souligné dans le vers 5 par les allitérations en $[\mathrm{m}]$ et en $[\mathrm{t}]$, et le renforcement des accents en fin d'hémistiches par le $[t]$ : «tout» / «mutile». L'inachèvement du poète devient manque radical, mutilation. Depuis son dénuement, c'est à une beauté violente et subversive qu'il se voue. 

et le début du vers 14 , valorisent la musicalité pour mettre en scène au cœur de la langue la dispersion et le ressenti dont parle le poète au vers 12 : les vers démultiplient les enjambements et les allitérations en [s] et [p], déplaçant les accents principaux de l'alexandrin vers 11 («prise... » et « sans... »), et jouant avec la répétition (avec «à ta paupière ») et la construction en miroir («disperse et ressens» et "pure et dérisoire ») : la matière sensible des mots s'affranchit de la structure. Mais j'ai voulu que la chute du poème brise ce mouvement. Le vers final reprenant la structure rythmique du premier vers du sonnet, déplore la victoire inéluctable du sens, mais aussi du prosaïsme, puisque "paupière lourde de sens » - est une agrégation de deux expressions idiomatiques. Le poème se clôt sur la pesanteur d'un sens auquel nous a ramené l'image de la paupière.

70 Après avoir donné ces quelques éléments d'analyse, et avant de laisser la parole à Amélie Derome, je voudrais sur ce thème de la mutilation et plus généralement de la blessure : ce thème traverse de part en part mon livre. Il le traverse non pas en raison de l'hémophilie historique du poète (proclamé avec splendeur par Baudelaire : «Poëte, notre sang nous fuit par chaque pore » dans " $\mathrm{A}$ Théodore de Banville ${ }^{55}$ »), mais parce qu'il me semble que la vulnérabilité est la condition de la sensibilité. C'est une idée que je dois à la lecture d'Emmanuel Lévinas ${ }^{56}$. La sensibilité exige des êtres de chair et de sang, qui puissent être blessés, qui puissent être atteints. Mon idée est ainsi que la poésie nous ramène en dernière instance à cette possibilité de la blessure, qui fait que nous ne sommes ni tout à fait des dieux ni tout à fait des morts. Elle est l'arme qui nous rend à notre chair et notre incarnation.

71 Simon Jardin 


\section{La traduction d' « Eye Liner »}

72 Contrairement à ce que son titre emprunté à l'anglais pourrait laisser entendre, la traduction d' «Eye liner » fut semée d'embûches. Le prolongement de la rime en [ã] tout au long du poème, ainsi que l'attention portée aux effets sonores et les nombreux bouleversements rythmiques constituaient autant de difficultés à relever.

Ma première version du poème témoigne d'une certaine résignation. La rime annexée en «ni île" restreignait mes possibilités de traduction dès les premiers vers. La traduction la plus fidèle du nom commun « île », island, me privait de toute possibilité de restituer cet effet. Je ne pus trouver aucun mot anglais commençant par «land» permettant de relancer le deuxième vers tout en préservant son sens. J'optais donc pour le diminutif islet, petite île, qui se transformait en I let, je laissais, au vers suivant. J'obtenais ainsi :

Recalling you: no voice, no warmth nor islet

(I let, when twenty, my sensations dwindle).

Ma difficulté à construire le troisième vers sur ces fondements aurait dû me pousser à emprunter une autre voie, mais je m'engouffrais pleinement dans cette impasse et me contentais d'une rime partielle et d'une inversion, procédé qu'évite pourtant généralement Simon Jardin et qui donne une coloration désuète au texte : "Could ever the dim conscience violate ». Le quatrième vers rompait franchement avec le sens de l'original, et j'osais un : " Of incompletion mine which I rekindle », c'est-à-dire, de mon inachèvement avec lequel je renoue, faisant fi de la loi générale de l'auteur qui devait me sembler alors bien lointaine, tout occupée que j'étais à préserver, coûte que coûte, la rime annexée. La règle particulière, la lettre, m'animait seule : l'esprit et la règle d'ensemble m'échappaient entièrement. Je m'attelai sereinement au second quatrain, abusant encore de ma nouvelle trouvaille: les rimes partielles. Nous trouvons ainsi mutilates au premier vers, qui rime vaguement avec violate, mais certainement pas avec islet, et driblets au troisième, rimant à peu près avec islet mais nullement avec violate. Mes rimes partielles devenaient chiasmatiques et je ne m'en préoccupais guère. Quant à la rime en «indle » promise, je la convertissais sereinement en une pseudo-rime en « ondled» ou « undled». Quand vint le moment de traduire les tercets, je pris le parti de m'affranchir définitivement des lois fixées par l'auteur et abandonnait la rime en [ã], que ma traduction faisait déjà passer de [et], à [ets] et de [ate] à [ates] et penchai pour une rime autonome et improvisée en [ing]. En exécutant cette traduction, je n'avais pas vu que j'avais également exécuté l'original.

75 J'ai, bien entendu, un peu exagéré mon portrait de traductrice sans foi ni loi dans ce bref commentaire. Ce dernier visait surtout à montrer la difficulté réelle qui résidait dans la traduction de ce poème. Ce n'est qu'au prix de nombreuses versions - et grâce aux commentaires de Simon Jardin - que je suis parvenue à en obtenir une traduction plus fidèle. Le problème demeurait cependant le même : je tenais à rendre aussi bien la rime annexée que la rime commune aux quatrains et aux tercets. Islet s'étant révélé inopérant, je pris le parti de m'éloigner un peu plus du sens du mot île, et choisis de traduire par reef, qui désigne un atoll. Je pus donc poursuivre en traduisant par « Rethink of when my senses were not five », qui parait certes éloigné du vers original mais qui restitue la présence d'un chiffre et qui désigne bien l'anesthésie des sentiments à laquelle Simon Jardin faisait référence. La nouvelle rime en " eef »me permit de traduire le troisième vers sans recourir à l'inversion : «Could ever tear away 
my dim belief ». Je repris enfin le quatrième vers de manière plus fidèle, obtenant : «In you: the incompletion I contrive! » et parvins à rendre les rimes en [ive] et [eef] dans le second quatrain. Les choses se compliquèrent pourtant aux tercets: survivre ne pouvait être traduit que par survive. Il me fallait donc remanier légèrement le schéma des rimes, et placer la rime en «ive " aux premier, deuxième et cinquième vers. Je souhaitais également transmettre quelque chose de la concession qui opposait survivre et délivre en français. Dans la mesure où aucun des synonymes de free ne rimait avec survive, j'ai décidé de recourir au contre rejet «And I've ». Le placement du pronom en fin de vers me semblait ainsi insister sur le libre arbitre du poète. Il ne me restait plus qu'à trouver une dernière rime pour les vers restants, ici en « id ». Bref, la traduction complète du poème me paraît avoir découlé du respect d'un seul effet, celui de la rime annexée et cet exemple montre combien, en traduction, l'application de la plus petite loi entraîne une série de lois supplémentaires en cascade.

Les nombreuses allitérations qui parcourent le poème constituaient une règle additionnelle. La traduction du vers "Qui seul a su mêler ma lutte à l'inutile" représentait le double défi de trouver des mots suffisamment longs pour qu'ils puissent former un pentamètre et qui témoignent en même temps d'une parenté de sonorités. J'avais d'abord songé à associer trifle, vétille, et strife, lutte. Hélas, je ne parvins à les combiner qu'au prix du sens du vers et d'un ajout qui permettait de récupérer la rime, ce qui donnait : "To mix what's trifle with strife's leitmotif». Si les allitérations étaient bien au rendez-vous, l'inclusion du terme à forte connotation leitmotif contrevenait au sens original. Je trouvais enfin "To mix my strife with what was never chief", présentant des allitérations en [w] et en [f]. S'imposer de traduire un effet, c'est accepter que le texte traduit en produise de nouveaux.

La traduction de la musicalité des quatre derniers vers demeure sans doute ce qui me paraît le mieux éclairer ce phénomène. Simon Jardin m'avait en effet demandé de prêter une attention particulière aux différents effets de rythme et de sonorités. J'ai ainsi tâché de restituer le décalage des accents en français, quoique ma version ne les situe pas nécessairement aux mêmes endroits. Afin de rendre la construction en 5/7 du vers "D'entre les rêves, prise à ta paupière sans ", j'ai choisi de placer trois syllabes accentuées à la suite les unes des autres «Amid dreams, seized within your eyelid». Les allitérations en [p] et [r] se muent en allitération en [d] et assonances en [i]. Une rime interne en [Id], amid et eyelid, rappelle la présence du son [ã] au début et à la fin du vers français. Le contre-rejet "sans" est déplacé, comme je l'ai déjà indiqué, au vers suivant. L'allitération en [d] s'y prolonge également sowed et rid, et le vers amorce une allitération en [s], entamée au vers précédent par seized, avec les mots solace et sowed. Enfin, le dernier vers reprend le procédé de la rime interne lid et loaded.

78 L'ultime vers du sonnet fut d'ailleurs d'autant plus délicat à traduire fidèlement qu'il résultait de l'agrégation de deux locutions idiomatiques, " paupière lourde » et « lourd de sens. » Instinctivement, il me semblait que la traduction d'une expression courante ne pouvait se faire aisément, et je me mis en quête d'images tout autres. Je tentai d'abord un jeu sur le sens de socket, qui désigne aussi bien l'orbite de l'œil que la prise de courant :

The idle nerve of living on the hook

of sockets yours, whose current comes from meaning. 
Le poète vivait donc bien à l'orbite de l'être aimé, dont le courant électrique provenait du sens. Le sens de l'original, quant à lui, se trouvait quelque peu dissipé dans la double image. Je proposais ensuite :

I feel and sow of living on the hook

of your eyelash, sharp as whip alas!

qui se fondait sur le double sens de lash, cil ou bien fouet. Cette fois-ci, le problème se nichait dans l'impossibilité de reprendre la rime en lash ou hook dans les quatrains. La meilleure solution, finalement, fut la plus littérale :

of the inane impertinence to thrive

Against your lid: already heavy loaded.

81 Cette étude de ma traduction d' "Eye liner » me paraît montrer la part de risque que comporte chaque traduction. La restitution d'effets formels conduit rarement à la construction d'une équivalence parfaite entre le texte traduit et le texte original, mais résulte plutôt de la disparition des effets de l'original, compensée par la production de nouveaux effets analogues. Ainsi, la traduction procède bien de cette entreprise d'altération de l'original que ses détracteurs lui reprochent. La métaphore de la traduction comme blessure portée au corps du texte se file en effet au long des siècles. Le préfacier des Voyages de Gulliver de 1838 accusait ainsi les premiers éditeurs français de l'œuvre d'avoir commis «trop de négligences, trop d'inexactitudes, trop de mutilations graves ${ }^{57}$ ", ce qui le poussait naturellement à publier une nouvelle traduction. En suivant le nouveau traducteur de Gulliver, nous serions tenté de conclure qu'il existerait de bonnes traductions - les traductions intègres - et de mauvaises traductions - les traductions mutilantes. Cette vision me parait toutefois quelque peu naïve, dans la mesure où la mutilation relève, en quelque sorte, du crime fondateur de la traduction : c'est-à-dire qu'elle forme le prix à payer pour que le texte original puisse exister en langue étrangère. Les réserves que l'on pourrait émettre à cet égard reposent sur la trahison de la pensée de l'auteur. Or, dans le cas qui nous occupe aujourd'hui, cette méfiance ne semble pas avoir lieu d'être, puisque l'auteur a sanctionné les choix opérés tout au long du processus de traduction. La mutilation devient ainsi l'épreuve que l'original doit subir pour coaguler dans une autre langue. Refuser ce risque reviendrait à refuser au texte original toute condition de survie.

\title{
Étude de cas 2, « Ne plus se pardonner... » : blesser peut être une règle
}

\author{
Ne plus se pardonner de vivre \\ Un soir à soi évoquer ivre \\ L'illusion de se réparer \\ Sans disposer d'outil valable \\ Hormis ces nudités instables \\ Et ces miroirs défigurés, \\ Lorsque plus rien ne nous délivre \\ Et névralgie au cœur du givre, \\ Plus rien ne nous semble assuré, \\ Que nos destins deviennent fables \\ Et vu du ciel cibles et sables \\ Dans un viseur décoloré,
}


Le sens est perdu de survivre,

On ne meurt pas que dans les livres,

L'animal en nous égaré

Vient flairer en dessous des tables

Le jour qui vient, l'inéluctable

Où ses os seront déterrés.

83

\section{Simon Jardin}

Amends for living yet unmade

One night, apart, a thirst conveyed

Imagination of repair

Without a proper tool in sight

Except the naked bodies blight

Which crooked mirrors do compare,

When hopes of freedom fully strayed,

Neuralgia into frost forayed

And what seemed real slipped in thin air,

May fates be fables we recite

And sands and targets seen in flight

At gunpoint tarnish unaware,

Survival sense no longer made

Death in books is life's own trade

The stranded beast in our hearts' lair

Sniffs under tables in a fright

The day to come, the certain plight

When its old bones will be laid bare.

Le vers 1 s'ouvre sur un constat de culpabilité : « Ne plus se pardonner de vivre ». Vivre est conçu comme une faute; ce qu'on peut rapprocher du thème du péché originel, mais aussi de la culpabilité du survivant. Ce constat place immédiatement le livre sous le signe de la faute et du malaise. L'infinitif utilisé pour ce constat se retrouve dans le reste du quatrain, bien que soient décrites les émotions d'un individu, l'impersonnalité maintenue au vers 2 avec «soi » laisse au lecteur une place pour s'approprier cette 
émotion. Mais en même temps cette impersonnalité souligne l'idée que la culpabilité de vivre rend celui qui la porte étranger à lui-même.

Le vers 2 temporalise et situe: "Un soir à soi ». Ainsi l'émotion (v. 1) précède la situation (v. 2), la faute précède la situation. Ce début de vers mérite qu'on s'y attarde, puisqu'il donne lieu à un jeu paronomastique entre "soir » et "soi » renforcé par le rythme iambique. Cela induit une équivalence entre le « soir » et le » soi » qui donne au soi une teinte vespérale, crépusculaire. De plus, de "soir " à "soi», une lettre est perdue : le soi est pensé comme manque et comme inachèvement, prolongeant l'idée d'un être étranger à lui-même. Avec l'infinitif, c'est de manière impersonnelle qu'est décrite cette situation de solitude et d'isolement. La deuxième partie laisse place une rupture sur le rythme iambique : c'est la lancinance de l'ivresse qui s'installe, le mot « ivre » placée sur la dernière syllabe et le dernier accent arrive presque comme un hoquet.

90 Le vers 3 explicite l'évocation : elle n'est pas heureuse, mais elle désigne un espoir en même temps qu'une impossibilité. L'illusion de la réparation se rapporte à la faute initiale, comme un rachat, mais c'est aussi une réification de l'individu. L'image d'une humanité brisée et irréparable se fait jour ici.

91 Le vers 4 commençant par «sans » prolonge ce qui devient un véritable champ lexical de la déficience et du dénuement. L'impossibilité désigne alors une inadéquation des moyens que l'individu a à sa disposition.

Le vers 5 et le vers 6 viennent prendre le contrepied de cette impuissance d'une manière ambiguë: les seuls outils de la réparation sont décevants et peu fiables, «nudités instables » et « miroirs défigurés » : la chair destinée à pourrir et les visages qu'on ne reconnaît plus. Ces nudités qui semblent insaisissables, à peine réelles (des êtres qui se déterminent une qualité abstraite) et ces miroirs qui prennent la mutilation des visages qui s'y reflètent (des objets qui sont plus vivants que les êtres) : ce sont deux réalités contemporaines, la pornographie et les écrans qui la supportent. Ce sont en même temps les seuls outils qu'il reste parce que les unes nous renvoie à autrui et les autres nous renvoient à nous-mêmes. Ils invitent à la rencontre des corps ou à l'examen de conscience.

93 La fin de la strophe semble hésiter sur la possibilité d'une reconnaissance : les miroirs défigurés décriraient-ils la condition du poète, condamné à ne plus reconnaître son reflet, pour que le lecteur puisse s'y projeter?

\section{Deuxième sizain - vers 7 à 12}

94 C'est alors que le poète opère une rupture dans l'impersonnalité : le « nous » des vers 7 et 9 désigne une condition partagée. Le poète inclut le lecteur : nous sommes dans le même sac. Les vers 7 et 9 explicite cette condition. Le ton devient oratoire, cesse d'être intimiste. $\mathrm{Au}$ «ne plus » du premier sizain, répond la répétition des "plus rien » qui souligne l'absence de délivrance et l'absence de fondement qui caractérise la condition négative du poète et du lecteur. Le vers 6 vient en avance caractériser le vers 7, ce qui produit un premier effet de suspens : la «névralgie» explicite l'absence fondement puisqu'elle est une poussée de douleur dont la cause est dans la plupart des cas non identifiée. L'image complète : « névralgie au cœur du givre » continue l'hésitation entre humain et inhumain : la poussée de douleur se produit au cœur du givre. Elle évoque 
une souffrance qui glace et se cristallise, alors que la paronomase déploie les sons de «névralgie » dans ceux du mot « givre ».

Le vers 10 débute une série de métaphores qui viennent qualifier le destin des êtres sans délivrance et sans fondement. Le destin devient une fable, quelque chose qui relève de la fiction, voire du mensonge. La fable est en même temps un genre littéraire qui nous enseigne la réalité dans sa dureté et son âpreté, avec ses morales souvent cruelles.

La métaphore évolue au vers 11 avec un changement d'angle de caméra "vus du ciel ", le destin nous échappe tellement que le poème procède une véritable coupe cinématographique: le plan est désormais zénithal. Ces plans sont de nos jours effectués à l'aide de drones. L'ambiance du poème devient militaire. Les destins sont pris pour cible et pris pour sable. La hauteur du point de vue crée une disproportion: les hommes sont grains de sable. La paronomase induit l'idée qu'ils sont cibles parce que sables, sables parce que cibles.

Le vers 12 explicite la métaphore et l'ambiance: les viseurs décolorés sont ceux de l'aviation et des drones militaires. La décoloration renvoie aux images infra-rouges qui y sont associées. Le "viseur décoloré » marque un retour à l'impersonnel : le ciel se fait menace. Le dieu personnel s'est absenté de ce ciel traversé par les machines.

$\mathrm{Au}$ vol stationnaire de ces machines de morts et d'images, se combine l'effet de suspens et de ralenti de l'ensemble de ce sizain composé par des propositions subordonnées dont on attend la principale qui ne viendra qu'à la strophe suivante.

\section{Troisième sizain - vers 13 à 18}

Le dernier sizain produit une accélération soudaine avec l'arrivée de la principale au vers 13 et le vers 14 qui s'enchaîne. De plus, si l'on observe la manière dont sont construites les strophes précédentes, on remarque qu'ils sont scindés en deux groupes de trois vers. Le dernier fait rupture puisqu'il est structuré par un groupe de deux vers et un dernier groupe de quatre vers.

Les vers 13 et 14 se présentent sous la forme de maxime qui rappelle la fable de la strophe précédente. Le premier vers est construit avec une inversion qui crée une ambiguïté : il peut signifier le sens de la survie est perdu ou bien la survie est la raison pour laquelle le sens est perdu. Or cette ambiguïté doit être prise au sérieux puisqu'elle produit à l'intérieur du langage lui-même la perte de sens dont parle le poème. Ce premier vers du sizain doit être mis en regard avec le premier du poème : on est passé de la culpabilité de vivre à l'absurdité de survivre. Du mot vivre au mot survivre, le poème fait alors appel à une dimension de l'homme qui n'est plus tout à fait humaine, mais déjà animale.

101 Le vers 14 éclaire d'une autre manière l'idée du vers précédent. Les livres donnent sens à nos morts, mais nous mourrons dans la réalité. Ce vers est construit comme une maxime issue de la sagesse populaire avec le «on» impersonnel qui vient contrebalancer le côté littéraire et soutenu du vers 13. Les deux vers se confirment et se complètent, tout en s'opposant dans le ton. Registre soutenu et registre populaire sont rangés dans le même sac.

Le vers 15 ouvre la fin du poème. Le thème de la survie renvoie désormais explicitement à l'animal en l'homme. La fable préparait ce thème, elle qui a souvent 
recours à des animaux personnifiés. Ce vers doit être mis en regard du vers 13 puisqu'il reprend le même procédé de construction et le même effet d'ambiguïté. "En nous " dont la position centrale dans le vers évoque un enfermement, une captivité, se rapporte aussi bien à «l'animal » qu'à «égaré »: le vers peut signifier: notre part animale qui n'existe plus, ou bien notre part animale qui erre. L'ambiguïté induit l'idée d'une espèce menacée.

Le vers 16 fait écho à l'ivresse du vers 2 du poème avec «en dessous des tables ». L'image finale continue de se construire: l'animal est désormais sorti de nous. Prolongeant les aller- retours entre humain et inhumain, l'homme s'efface derrière l'animal qu'il est : le voilà qui flaire en dessous des tables.

Le vers 17 opère un effet de suspens. Ce que flaire l'animal que nous sommes, c'est un moment inéluctable. Il y a un double mouvement de venue avec la répétition du verbe vers 16 et 17 : l'animal vient et le jour vient, l'un semblant appeler l'autre.

La résolution du poème arrive au dernier vers à la manière d'une chute. L'animal en nous flaire sa propre mort : nous flairons notre propre mort. Le modèle de l'animal est ainsi le chien qui aime à déterrer les os. Il y a là une réminiscence de de T.S. Eliott à la fin de « The Burial of the Dead» qui ouvre The Waste Land:

Oh keep the Dog far hence, that's friend to men,

Or with his nails he'll dig it up again ${ }^{59}$ !

L'humanité apparaît ainsi comme une espèce menacée, une espèce en: voie de disparition.

Simon Jardin

Si la version finale d' "Eye liner » fut le résultat de nombreux allers-retours et celle à laquelle j'ai consacré le plus de temps, la traduction de « Ne plus se pardonner » s'est déroulée de manière plus fluide. S'il m'est difficile de démêler les raisons de cette aisance relative, je vais tout de même me risquer à suggérer une piste possible. Je ne crois pas pouvoir attribuer ce phénomène à de mystérieuses affinités qui me relieraient au poème. En effet, je mentirais si je disais que je le préférais à « Eye liner » : les deux textes « me parlent » tout autant. En revanche il me faut reconnaître que ce poème, qui ouvre le recueil de Simon Jardin, est également le premier que j'ai lu de lui. Il m'était sans doute ainsi plus familier au moment de la traduction - j'avais eu, d'une certaine manière, le temps de le laisser reposer.

Dans la mesure où les questions formelles de traduction se sont résolues avec un certain naturel - les premières rimes que j'ai trouvées furent les bonnes - je privilégierai cette fois-ci les commentaires portant sur le sens du texte. La traduction des groupes verbaux fut l'un des points de départ de ma transposition. L'anglais ne me semblait pas permettre une traduction littérale du premier vers sans verser dans une forme de maladresse. La combinaison de l'impératif, du négatif et d'une forme pronominale s'accompagnait en effet d'une certaine lourdeur et aurait donné quelque chose de cet ordre "To stop forgiving ourselves to live ", formule nébuleuse et peu élégante Je décidai donc d'opter pour une forme passive, propre à transmettre l'impersonnalité qu'évoquait Simon Jardin: «Amends for living yet unmade.» Ce choix impliquait ainsi une rime en [ade], portant en germe de nombreuses répercussions sur la traduction des temps, dans la mesure où cette terminaison se trouve plus souvent au prétérit et au passif qu'à l'infinitif. Les infinitifs originaux des premiers vers de chaque strophe furent remplacés par des tournures passées ou passives. « Lorsque plus rien ne 
nous délivre " devint "When hopes of freedom fully strayed ", et «Le sens est perdu de survivre" "Survival sense no longer made". Si cette solution paraissait contrevenir à l'aspect déclamatoire du poème, elle permettait toutefois d'en faire la fable évoquée au quatrième vers de la deuxième strophe, ce qui me semblait compenser la perte relative.

Cette traduction me paraît également soulever la question des interpolations et de leurs effets. Elle comprend en effet plusieurs ajouts qui ne figurent pas dans l'original, mais qui se sont avérés indispensables afin de retrouver la rime et de compléter les tétramètres. Ma traduction de «Sans disposer d'outil valable » allonge le texte français "Without a proper tool in sight ". Il m'aurait semblé dommage de remanier la traduction littérale "without a proper tool» dans la mesure où elle formait un trimètre iambique parfait. En ce sens, l'ajout de "in sight», en vue, me paraissait préférable à tout réaménagement $d u$ vers. L'introduction de cette locution permettait par ailleurs d'annoncer les miroirs qui apparaissent au dernier vers de la strophe - vers que la traduction allonge également. Je ne pouvais en effet traduire "défigurés» par disfigured. L'accentuation de l'adjectif ne m'autorisait pas de le placer dans un tétramètre iambique. Je recourais donc au synonyme crooked. Or, là où «miroir défigurés » représentent les trois quarts du vers français, «crooked mirrors » ne forme que la moitié du vers anglais. Je choisis donc d'immiscer la notion de comparaison, qui souligne les rapports des corps et des regards étrangers qu'évoquaient Simon Jardin. De la même manière, la traduction de "Que nos destins deviennent fables" ne pouvait s'accomplir sans ajout. La traduction littérale "May our fates become fables» ne constituait pas un vers régulier et ne suivait pas la rime imposée. Afin que le début du vers présente un iambe, il me fallait éluder le pronom et commencer par «may fates ». Il demeurait toutefois nécessaire d'introduire le pronom personnel "nous", caractéristique de la deuxième strophe originale. Je trouvais donc la possibilité suivante " May fates be fables we recite », qui, malgré l'ajout du verbe " réciter », renoue avec la rime et introduit le pronom souhaité tout en ne s'écartant pas de l'esprit du poème. Le dernier vers de la strophe présente également une nouveauté : l'adjectif unaware, qui signifie "sans le savoir». La seule traduction possible de «dans un viseur » était en effet « at gunpoint », puisque la version littérale « in gun sights » n'est que peu usitée. En complétant le vers avec l'idée de décoloration tarnish, on n'obtient que deux pieds et demi. J'ai donc tâché de trouver un adjectif qui correspondrait à l'esprit du vers et l'adjectif retenu me paraissait pouvoir qualifier l'infinitésimalité des hommes dont il était question dans l'original.

111 Contrairement à ce que j'indiquais tout à l'heure, j'avoue devoir céder, avant de conclure cette étude, à la tentation d'évoquer quelques exemples de réussite ou de déperdition relative d'effets formels. La paronomase de " Névralgie au cœur du givre " représentait l'un des enjeux formels majeurs du poème. Devant l'impossibilité d'une traduction littérale, je décidais de développer l'allitération à l'extérieur de neuralgia. Dans la version anglaise "Neuralgia into frost forayed ", la répétition $\mathrm{du}[\mathrm{r}]$ se redouble de l'accumulation des sons en [f] et en [o] dans les deux derniers mots du vers. Je ne parvins toutefois pas à restituer véritablement une deuxième paronomase, celle qui figure au vers que voici : "Un soir à soi évoquer ivre ", l'anglais ne permettant pas de parenté possible entre les termes désignant le soir et le soi. Je choisis de compenser cette perte par la combinaison de trois mots se terminant par la consonne [t]: night, apart et thirst. Enfin, la reprise du contraste opposant le registre populaire et le registre soutenu de l'ouverture du dernier sizain fut l'objet d'un soin minutieux. J'avais finalement ! - pour traduire le vers «Le sens est perdu de survivre» l'autorisation de 
recourir à l'inversion, qui a le mérite de faciliter la construction des vers réguliers anglais. J'obtins donc "Survival sense no longer made", qui préserve l'ambiguïté de l'original tout en respectant la forme. Il me fallait ensuite trouver une manière de rendre la sagesse populaire présente dans le vers suivant. La traduction littérale «We do not only die in books ", quoiqu'elle forme un tétramètre iambique, ne me paraissait pas remplir ce critère. Je choisis donc de recourir à la formule « Death in books is life's own trade ", dont le rythme trochaïque évoque la solidité de la maxime et la construction parallèle (trois et trois syllabes séparées par le verbe être) souligne l'idée originale du vers, selon laquelle les livres donnent sens à nos morts " death in books", mais que nous mourrons dans la réalité « life's own trade ».

L'étude de la traduction développe et complète les conclusions de notre première étude de cas. Nous constatons à nouveau que l'atteinte de la traduction d'un texte ne peut se passer de l'atteinte portée à ce dernier. Cette forme de mutilation, nouveau crime à ajouter à l'arsenal esquissé dans notre première partie, est pourtant la condition de la survie $\mathrm{du}$ texte. La traduction d'un poème rimé en vers régulier implique nécessairement certaines modulations du sens. Ces aménagements nous ont pourtant paru préférables à l'évacuation des questions formelles. Traduire la poésie en faisant abstraction de sa forme, n'est-ce pas la priver une seconde fois des armes dont la publicité et la chanson l'avait déjà dépouillée ? Ce qui n'a guère de sens, ce ne sont pas tant les légères oscillations de signification qu'impliquent la traduction de la forme, mais bel et bien les traductions qui refusent au poème son statut de poème. Ainsi, l'ambiguïté du vers «Le sens est perdu de survivre " parait éclairer l'ensemble de la démarche qui a guidé ma traduction de Voies de disparition. Faire survivre le poème, c'est-à-dire le faire exister en d'autres langues, implique une forme de déperdition du sens qu'il ne s'agit pas de lamenter, mais de compenser tout au long de la traduction. A l'inverse, le mépris de la traduction de la forme revient à faire perdre au texte toute survie possible. La traduction de la poésie a donc ceci de particulier qu'elle relève à la fois d'une mutilation, d'une amputation du texte, que de son extension, de sa survie.

Amélie Derome

114 Nous avons montré dans quelle mesure, de son exclusion à sa rébellion, le poète faisait figure de hors-la-loi. Le respect de la règle, loin d'en faire un serviteur de l'ordre, devenait alors outil pour subvertir et arme pour affronter; c'est dans ce cadre que j'inscrivais ma démarche. C'est alors que les crimes imputés à la traduction de la poésie vous ont été exposés; le traducteur prenait tour à tour le masque du vagabond et du clandestin, du pilleur et du contrebandier, du voleur et du violeur. Plus encore : en se plaçant du côté du poète, le traducteur devenait à son tour hors la loi ; c'était là la démarche d'Amélie Derome. Nous avons ensuite illustré notre propos en dégageant des règles générales pour mieux nous plonger dans le détail de deux poèmes et de deux traductions. Nous avons ainsi défendu l'idée d'une plaie originelle qu'il s'agissait d'assumer positivement, aussi bien dans la poésie que dans sa traduction :

So well thy words become thee as thy wounds;

They smack of honour both ${ }^{60}$.

Simon Jardin 


\section{BIBLIOGRAPHIE}

AKHMATOVA, Anna, Requiem, tr. Paul Valet, Paris, Éditions de Minuit, 1966

ANDROSENKO, Lilia, « Fiodor Dostoïevski traducteur de Balzac : vers la révélation d'un futur talent littéraire », in Itinéraires, 2, 2018, en ligne, consulté le 13 septembre 2019, URL : http:// journals.openedition.org/itineraires/4582

BAUDELAIRE, Charles, «La Genèse d'un poème ", in Edgar Allan Poe, Histoires grotesques et sérieuses, Paris, Michel Lévy Frères, 1871

- « A Théodore de Banville », in Les Fleurs du mal, Paris, Michel Lévy Frères, 1868, p. 108.

DU BELLAY, Joachim, La Défense et illustration de la langue française, Paris, Sansot, 1904

BERMAN, Antoine, L'Épreuve de l'étranger. Culture et traduction dans l'Allemagne romantique, Paris, Gallimard, 1984

BONNEFOY, Yves, « Traduction inédite de deux poèmes de John Donne : 'A hymne to Christ, at the Authors last going into Germany', 'Hymne to God my God, in my sicknesse' » in Palimpsestes, 2, 1990, p. 2-5.

BRODSKY, Jospeh, Poèmes 1961-1987, tr. Michel Aucouturier, Jean-Marc Bordier, Claude Ernoult, Hélène Henry, Ève Malleret, André Markowicz, Georges Nivat, Léon Robel, Véronique Schiltz et Jean-Paul Sémon, Paris, Gallimard, 1987

CELAN, Paul, Pavot et mémoire, tr. Valérie Briet, Paris, Christian Bourgois, 1987

- Grille de parole, tr. Martine Broda, Paris, Points, 2008

- La Rose de personne, tr. Martine Broda, trad. Martine Broda, Paris, Points, 2007

- Renverse du souffle, tr. Jean-Pierre Lefebvre, Paris, Points, 2006

CORBIERE, Tristan, Les Amours Jaunes, Paris, Glady, 1873

ELIOT, T. S., "The Burial of the Dead", in The Waste Land, in The New Anthology of American Poetry: Modernisms, New Brunswick, Rutgers University Press

ELLRODT, Robert, « Comment traduire la poésie ? » in Palimpsestes, Hors-série, 2006

FROST, Robert, « Conversations on the Craft of Poetry », in Elaine Barry, Robert Frost on Writing, New Brunswick, Rutgers University Press, 1973

GACOIN LABLANCY, Pauline, BASTIEN-THIRY, Adèle, « André Markowicz et les enjeux de la retraduction », in Bulletin de l'Institut Pierre Renouvin, 40, 2014, p. 83-94.

GENET, Jean, « Le Condamné à mort », in CEuvres complètes, vol. 2, Paris, Gallimard, 1951

HOMERE, The Iliad, tr. Pope, Londres, W. Bowyer, 1715

- Iliade, tr. Anne Dacier, Paris, Guérin 1761

JARDIN, Simon, Voies de disparition, Paris, Éditions du Cerf, 2019

- Nous Resterons Inconsolés, court-métrage, Paris \& Hong-Kong, The Empire Company Ltd, 2019

LACENAIRE, Pierre-François, " Chant de mort », in Mémoires, révélations et poésies de Lacenaire, vol. 2, Paris, Marchands de nouveautés, 1836, p. 140.

LADMIRAL, Jean-René, Sourcier ou cibliste, Les Belles Lettres, Paris, 2014 
LARBAUD, Valéry, Sous l'invocation de Saint Jérôme, Paris, Gallimard, 1997

LEVINAS, Emmanuel, Humanisme de l'autre homme, Paris, Paris, Le Livre de Poche, 1987

MANDELSTAM, Ossip, Poèmes et essais, tr. Christian Mouze, Paris, Alidades, 1987

- Poèmes, tr. Tatiana Roy, Paris, L'Âge d'homme, 1984

- Les Cahiers de Voronej (1935-1937), tr. Henri Abril, Strasbourg, Circé, 1998

- Les Poésies d'amour, tr. Henri Abril, Strasbourg, Circé, 1984

MARKISH, Peretz, Anthologie de la poésie yiddish, tr. Charles Dobzynski, Paris, Gallimard, 2000

MAROT, Clément, « Chanson III », in L'Adolescence clémentine, Paris, Antoine Bonnemère, p. 372.

PESSOA, Fernando, Le Gardeur de Troupeau, Poésies d'Alvaro de Campos, tr. Armand Guibert, Paris, Gallimard, 1987

- Poèmes Anglais, tr. Georges Thinès, Paris Editions Points, 2011

POE, Edgar, «Le Corbeau », tr. Baudelaire, in Histoires grotesques et sérieuses, Michel Lévy frères, Paris, 1871

RILKE, Rainer Maria, Élégies de Duino, Les Sonnets à Orphée, tr. Roger Lewinter, Paris, Gérard Lebovici, 1989

- Huitième élégie de Duino, tr. Roger Munier, Paris, Fata Morgana 1998

- Poèmes à la nuit, tr. Gabrielle Althen et Jean-Yves Masson, Lagrasse, Verdier, 1994

SHAKESPEARE, William, Macbeth, in Complete Works, London, Oxford University Press, 1955

- Macbeth, tr. François-Victor Hugo, Euvres complètes, Paris, Pagnerre, 1866

STEINER, George, Après Babel : une poétique du dire et de la traduction, Paris, Albin Michel, 1998

SWIFT, Jonathan, Voyages de Gulliver dans des contrées éloignées, La Haye, Pierre Gosse et Jean Neaulme, 1727

- Voyages de Gulliver, tr. Pierre-Guyot Desfontaines, Paris, Guérin, 1727

- Voyages de Gulliver, Paris, Furne et Fournier, 1838

TSVETAIEVA, Marina, Le Ciel brûle, Paris, tr. Pierre Léon et Ève Malleret, Paris, Gallimard, 1999

VALERY, Paul, « Abattage d'un arbre », Commerce, XIV, Paris, 1927

VENUTI, Lawrence, The Translator's Invisibility. A History of Translation. London and New York, Routledge, 2002

VERLAINE, Paul, «Chanson d'automne », in Poèmes saturniens, Vanier, 1902, p. 34.

VILLION, François, « Ballade des pendus », in Euvres de Maistre François Villon, Paris, Ebrard, 1835

DE WAILLY, Henri, « Traduttore, traditore », in Les Cahiers de la publicité, Paris, 17, 1967

« ‘А ВЫ УЧИЛИСЬ ЭТОМУ?’ СТЕНОГРАММА СУДА НАД ИОСИФОМ БРОДСКИМ », (« 'Vous a-t-on appris à faire cela ?' Transcription du procès de Joseph Brodsky »), TV Train, 12 mars 2014, en ligne, consulté le 1 septembre 2019, URL : https://tvrain.ru/teleshow/archive/

a_vy_uchilis_etomu_stenogramma_suda_nad_iosifom_brodskim-378906/ 


\section{NOTES}

1. «La poésie est ce qui se perd à la traduction », notre traduction. Robert Frost, « Conversations on the Craft of Poetry ", in Elaine Barry, Robert Frost on Writing, New Brunswick, Rutgers University Press, 1973, p. 159.

2. Dante, cité par Robert Ellrodt, "Comment traduire la poésie?» in Palimpsestes, Hors-série, 2006, p. 1.

3. Edgar Poe, «Le Corbeau », tr. Baudelaire, in Histoires grotesques et sérieuses, Michel Lévy frères, Paris, 1871.

4. Homère, The Iliad, tr. Pope, Londres, W. Bowyer, 1715.

5. Charles Baudelaire, "La Genèse d'un poème", in Edgar Allan Poe, Histoires grotesques et sérieuses, Paris, Michel Lévy Frères, 1871, p. 337.

6. Paul Valéry, "Abattage d'un arbre", Commerce, XIV, Paris, 1927, p. 5-9. Yves Bonnefoy, «Traduction inédite de deux poèmes de John Donne: 'A hymne to Christ, at the Authors last going into Germany', 'Hymne to God my God, in my sicknesse' » in Palimpsestes, 2, 1990, p. 2-5.

7. Ellrodt, op. cit., p. 14.

8. Simon Jardin, Voies de disparition, Paris, Éditions du Cerf, 2019.

9. Tristan Corbière, Les Amours Jaunes, Paris, Glady, 1873, p. 4.

10. Voir à ce sujet « ‘А ВЫ УЧИЛИСЬ ЭТОМУ?' СТЕНОГРАММА СУДА НАД ИОСИФОМ БРОДСКИМ », ( 'Vous a-t-on appris à faire cela ?' Transcription du procès de Joseph Brodsky »), TV Train, 12 mars 2014, en ligne, consulté le 1 septembre 2019, URL: https://tvrain.ru/teleshow/archive/ a_vy_uchilis_etomu_stenogramma_suda_nad_iosifom_brodskim-378906/.

11. François Villon, «Ballade des pendus », in Euvres de Maistre François Villon, Paris, Ebrard, 1835, p. 306.

12. Jean Genet, «Le Condamné à mort ", in Euvres complètes, vol. 2, Paris, Gallimard, 1951, p. 215.

13. Pierre-François Lacenaire, "Chant de mort », in Mémoires, révélations et poésies de Lacenaire, vol. 2, Paris, Marchands de nouveautés, 1836, p. 140.

14. Henri de Wailly, « Traduttore, traditore ", in Les Cahiers de la publicité, Paris, 17, 1967, p. 22.

15. Antoine Berman, L'Épreuve de l'étranger. Culture et traduction dans l'Allemagne romantique, Paris, Gallimard, 1984.

16. Id.

17. Voir à ce sujet: Pauline Gacoin Lablancy, Adèle Bastien-Thiry, "André Markowicz et les enjeux de la retraduction », in Bulletin de l'Institut Pierre Renouvin, 40, 2014, p. 83-94.

18. Voir l'article de Lilia Androsenko, «Fiodor Dostoïevski traducteur de Balzac: vers la révélation d'un futur talent littéraire ", in Itinéraires, 2, 2018, en ligne, consulté le 13 septembre 2019, URL : http://journals.openedition.org/itineraires/4582.

19. «[...] qui peut souffrir que des Princes préparent eux-mêmes leurs repas ; que les fils des plus grands rois gardent les troupeaux, qu'ils travaillent eux-mêmes, et qu'Achille fasse chez lui les fonctions les plus serviles ? » Homère, Iliade, tr. Anne Dacier, Paris, Guérin 1761, p. XXIV-XXV.

20. "Pratique culturelle dissidente", notre traduction. Lawrence Venuti, The Translator's Invisibility. A History of Translation. London and New York, Routledge, 2002, p. 125.

21. "L'esthétique dominante de la situation réceptrice », notre traduction. Ibid., p. 252.

22. «Un nomade dans sa propre langue, l'anglais, un fugitif au sein de sa langue maternelle», notre traduction. Id.

23. Joachim du Bellay, La Défense et illustration de la langue française, Paris, Sansot, 1904, p. 176.

24. Ibid., p. 177.

25. Valéry Larbaud, Sous l'invocation de Saint Jérôme, Paris, Gallimard, 1997, p. 69.

26. Comparer par exemple les traductions de Gulliver's Travels imprimées à La Haye et à Paris en 1727. Jonathan Swift, Voyages de Gulliver dans des contrées éloignées, La Haye, Pierre Gosse et Jean 
Neaulme, 1727. Jonathan Swift, Voyages de Gulliver, tr. Pierre-François Guyot Desfontaines, Paris, Guérin, 1727.

27. Jean-René Ladmiral, Sourcier ou cibliste, Les Belles Lettres, Paris, 2014, p. 23.

28. Voir notamment George Steiner, Après Babel : une poétique du dire et de la traduction, Paris, Albin Michel, 1998.

29. Cf. les travaux de Roland Barthes ou de Hans Robert Jauss.

30. Simon Jardin, Nous Resterons Inconsolés, Paris \& Hong-Kong, The Empire Company Ltd, 2018.

31. Jardin, Voies de disparition, p. 9

32. Ibid., p. 16.

33. Ibid., p. 62.

34. Ibid., p. 66.

35. Ibid., p. 77.

36. Ibid., p. 78.

37. Ibid., p. 85.

38. Ibid., p. 116.

39. Ibid., p. 108.

40. Ibid., p. 103.

41. Ossip Mandelstam, Poèmes et essais, tr. Christian Mouze, Paris, Alidades, 1987, Poèmes, tr. Tatiana Roy, Paris, L'Âge d'homme, 1984, Les Cahiers de Voronej (1935-1937), tr. Henri Abril, Strasbourg, Circé, 1998,

Les Poésies d'amour, tr. Henri Abril, Strasbourg, Circé, 1984.

42. Rainer Maria Rilke, Élégies de Duino, Les Sonnets à Orphée, tr. Roger Lewinter, Paris, Gérard Lebovici, 1989, Huitième élégie de Duino, tr. Roger Munier, Paris, Fata Morgana 1998, Poèmes à la nuit, tr. Gabrielle Althen et Jean-Yves Masson, Lagrasse, Verdier, 1994.

43. Joseph Brodsky, Poèmes 1961-1987, tr. Michel Aucouturier, Jean-Marc Bordier, Claude Ernoult, Hélène Henry, Ève Malleret, André Markowicz, Georges Nivat, Léon Robel, Véronique Schiltz et Jean-Paul Sémon, Paris, Gallimard, 1987.

44. Peretz Markish, Anthologie de la poésie yiddish, tr. Charles Dobzynski, Paris, Gallimard, 2000.

45. Fernando Pessoa, Le Gardeur de Troupeau, Poésies d'Alvaro de Campos, tr. Armand Guibert, Paris, Gallimard, 1987, Poèmes Anglais, tr. Georges Thinès, Paris Editions Points, 2011.

46. Anna Akhmatova, Requiem, tr. Paul Valet, Paris, Éditions de Minuit, 1966.

47. Marina Tsvetaieva, Le Ciel brûle, Paris, tr. Pierre Léon et Ève Malleret, Paris, Gallimard, 1999.

48. Paul Celan, Pavot et mémoire, tr. Valérie Briet, Paris, Christian Bourgois, 1987, Grille de parole, tr. Martine Broda, Paris, Points, 2008, La Rose de personne, tr. Martine Broda, trad. Martine Broda, Paris, Points, 2007, Renverse du souffle, tr. Jean-Pierre Lefebvre, Paris, Points, 2006.

49. Simon Jardin, op. cit., p. 116.

50. Ibid., p. 77.

51. Ibid., p. 85.

52. Ibid., p. 16.

53. Ibid. p. 108.

54. Clément Marot, "Chanson III ", in L'Adolescence clémentine, Paris, Antoine Bonnemère, p. 372.

55. Charles Baudelaire, «A Théodore de Banville », in Les Fleurs du mal, Paris, Michel Lévy Frères, 1868, p. 108.

56. Voir notamment Emmanuel Lévinas, Humanisme de l'autre homme, Paris, Paris, Le Livre de Poche, 1987, p. 104.

57. Swift, Voyages de Gulliver, Paris, Furne et Fournier, 1838, p. 2.

58. Paul Verlaine, "Chanson d'automne ", in Poèmes saturniens, Vanier, 1902, p. 34.

59. T. S. Eliot, "The Burial of the Dead", in The Waste Land, in The New Anthology of American Poetry: Modernisms, New Brunswick, Rutgers University Press, p. 407. 
60. William Shakespeare, Macbeth, in Complete Works, London, Oxford University Press, 1955, p. 846-7. Tes paroles te vont aussi bien que tes blessures : - elles sentent également l'honneur. » Shakespeare, Macbeth, tr. François-Victor Hugo, Euvres complètes, Paris, Pagnerre, 1866, p. 75.

\section{RÉSUMÉS}

La poésie semble faire doublement figure de hors-la-loi dans le champ de la littérature contemporaine et la voix du poète paraît, aujourd'hui, condamnée à une forme d'exclusion. Simon Jardin considère en effet que les poètes des cinquante dernières années auraient été expropriés. Les moyens qui leur étaient propres paraissent avoir été confisqués, notamment par la chanson et la publicité. Les slogans se sont emparés de la rime et de la versification, deux procédés que le marché éditorial de la poésie contemporaine tend à rejeter et dont Simon Jardin se ressaisit. Les auteurs ayant recours aux règles de la poésie qui en formaient jadis la voie royale se voient ainsi relégués aux marges de leur art, accusés de faire fausse route. D'une manière similaire, la traduction des rimes et des vers demeure décriée par l'édition contemporaine. La méfiance envers les règles héritées de la poésie croît et les rimes sont peu traduites, de crainte que la traduction ne bascule vers l'adaptation. La suprématie est accordée à la langue d'arrivée et les aspérités du langage sont volontiers gommées. Le travail de traduction de Voies de disparition de Simon Jardin par Amélie Derome s'inscrit dans une démarche de fidélité à la forme des poèmes : rimes et vers sont traduits. Cet article vise à montrer la manière dont la transposition des règles formelles contrevient aux normes de l'édition contemporaine et tend à faire de la traduction de la poésie une activité marginale, sinon illicite.

In the contemporary French editorial field, poetry appears to be cast aside, and the poet's voice seems to be condemned to a form of exclusion. Simon Jardin indeed believes that poets have been expropriated over the past fifty years. Their very own means seem to have been confiscated by song writing and advertising. Slogans have taken hold of both rhyme and verse, two types of techniques which the contemporary editorial market tends to dismiss and which Simon Jardin attempts to claim again. The authors who resort to the rules of poetry which used to pave the way to success thus end up being relegated to the margins of their art and are blamed for going astray. In a similar fashion the translation of rhyme and verse remains decried by contemporary publishing houses. Suspicion regarding the inherited rules of poetry grows and rhymes are rarely translated, lest translation should tilt towards adaptation. The target language gains hegemony and all linguistic asperities are toned down. Amélie Derome's translation of Voies de disparition by Simon Jardin consists in an attempt at faithfully translating the poems' form: rhyme and verse are translated. This article aims at revealing the ways in which the transposition of formal rules represents a breech in contemporary editorial norms and tends to show how the translation of poetry can be a marginal or even an unlawful activity.

La poesía parece estar doblemente prohibida en el campo de la literatura contemporánea y la voz del poeta parece hoy condenada a una forma de exclusión. Simon Jardin considera que los poetas de los últimos cincuenta años han sido expropiados. Sus propios recursos parecen haber sido confiscados, en particular a través de canciones y publicidad. Los eslóganes se han apoderado de la rima y la versificación, dos procesos que el mercado editorial de la poesía contemporánea tiende a rechazar y de los que Simon Jardin se está recuperando. Los autores que utilizan las 
reglas de la poesía que una vez formaron el camino real son relegados a los márgenes de su arte, acusados de cometer un error. De manera similar, la traducción de rimas y versos sigue siendo criticada por la edición contemporánea. La desconfianza en las reglas heredadas de la poesía va en aumento y las rimas rara vez se traducen, a fin de que la traducción no se transforme en adaptación. La supremacía se da al idioma de destino y la aspereza del idioma se borra fácilmente. El trabajo de Amélie Derome sobre la traducción de las Voies de disparition de Simon Jardin forma parte de un proceso de fidelidad a la forma de los poemas: se traducen rimas y versos. Este artículo pretende mostrar cómo la transposición de las reglas formales contraviene las normas de la edición contemporánea y tiende a hacer de la traducción de poesía una actividad marginal, si no ilegal.

\section{INDEX}

Palabras claves : Poesía, traducción, verso regular, rima, Simon Jardin

Mots-clés : Poésie, traduction, vers régulier, rime, Simon Jardin

Keywords : Poetry, translation, regular verse, rhyme, Simon Jardin

\section{AUTEURS}

\section{AMÉLIE DEROME}

Amélie Derome est traductrice et doctorante contractuelle de littérature britannique à Aix Marseille Université. Sa thèse, « Les traductions françaises de Gulliver's Travels de Jonathan Swift (1727-2017) : entre errances et résonances ", est dirigée par Jean Viviès au LERMA. Elle a présenté ses recherches sur la réception des traductions de l'œuvre à Paris-Sorbonne en juin 2017 et lors de plusieurs séances du Séminaire $C$ du LERMA. Son article consacré à la traduction du paratexte de Gulliver's Travels au XVIII ${ }^{\mathrm{e}}$ siècle paraîtra en 2018 dans la revue Book Practices and Textual Itineraries aux PUN. Elle a également participé au Congrès 2018 de la SAES en évoquant les liens qui unissent la traduction et la révolution et est intervenue pendant la Conférence internationale Walter Scott de la même année. Elle s'est enfin impliquée dans plusieurs événements de médiation scientifique, dont la Nuit des Chercheurs de Marseille en septembre 2017 et les Jeunes Chercheurs dans la Cité à Lille et à Bruxelles en janvier et février 2018.

\section{SIMON JARDIN}

Simon Jardin est un poète français dont le recueil Voies de disparitions a été publié aux Editions du Cerf en 2019. Ancien élève de classe préparatoire littéraire au lycée Henri IV, il a obtenu un master de philosophie à l'Université Panthéon-Sorbonne (mention Très Bien). Ses deux mémoires, consacrés à Emmanuel Lévinas, ont été encadrés par Renaud Barbaras. Il est également réalisateur de sept courts-métrages autofinancés, dont cinq en duo avec Mario Valero ; il prépare désormais un film produit par Samuel Bourdin. 